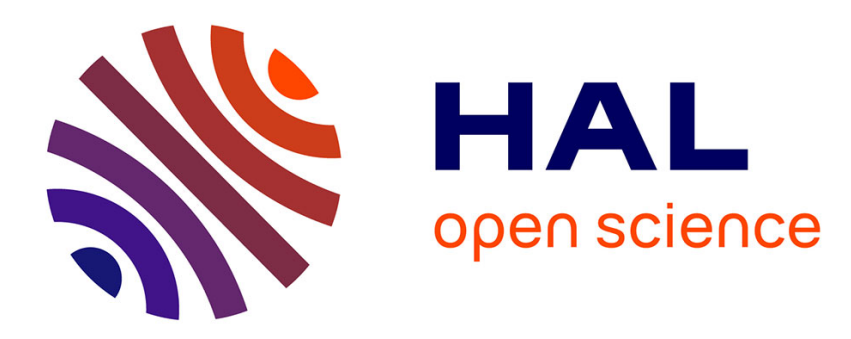

\title{
Singular perturbation of optimal control problems on multi-domains
}

Nicolas Forcadel, Zhiping Rao

\section{To cite this version:}

Nicolas Forcadel, Zhiping Rao. Singular perturbation of optimal control problems on multi-domains. SIAM Journal on Control and Optimization, 2014, 52 (5), pp.2917-2943. 10.1137/130916709 . hal00812846

\section{HAL Id: hal-00812846 https://hal.science/hal-00812846}

Submitted on 12 Apr 2013

HAL is a multi-disciplinary open access archive for the deposit and dissemination of scientific research documents, whether they are published or not. The documents may come from teaching and research institutions in France or abroad, or from public or private research centers.
L'archive ouverte pluridisciplinaire HAL, est destinée au dépôt et à la diffusion de documents scientifiques de niveau recherche, publiés ou non, émanant des établissements d'enseignement et de recherche français ou étrangers, des laboratoires publics ou privés. 


\title{
SINGULAR PERTURBATION OF OPTIMAL CONTROL PROBLEMS ON MULTI-DOMAINS*
}

\author{
NICOLAS FORCADEL ${ }^{\dagger}$ AND ZHIPING RAO
}

\begin{abstract}
The goal of this paper is to study a singular perturbation problem in the framework of optimal control on multi-domains. We consider an optimal control problem in which the controlled system contains a fast and a slow variables. This problem is reformulated as an Hamilton-JacobiBellman (HJB) equation. The main difficulty comes from the fact that the fast variable lives in a multi-domain. The geometric singularity of the multi-domains leads to the discontinuity of the Hamiltonian. Under a controllability assumption on the fast variable, the limit equation (as the velocity of the fast variable goes to infinity) is obtained via a PDE approach and by means of the tools of the control theory.
\end{abstract}

Key words. singular perturbations, optimal control, Hamilton-Jacobi-Bellman equations, essential Hamiltonians, multi-domains

AMS subject classifications. 49L25, 93C $73,35 \mathrm{~F} 25$

1 Introduction In the present work, we investigate a class of singular perturbation problems for Hamilton-Jacobi-Bellman equations motivated by optimal control systems with different time scales on multi-domains. The multi-domains considered here is the following repartition of $\mathbb{R}^{2}$ by two disjoint open subsets $\Omega_{1}, \Omega_{2}$ with

$$
\mathbb{R}^{2}=\bar{\Omega}_{1} \cup \bar{\Omega}_{2}, \Omega_{1} \cap \Omega_{2}=\emptyset .
$$

Consider the nonlinear controlled systems of the following form: given the final time $T>0$ and the initial data $t \geq 0, x \in \mathbb{R}^{d}, y \in \mathbb{R}^{2}$,

$$
\begin{cases}\dot{X}(s)=f(X(s), Y(s), \alpha(s)) & \text { for } \alpha(s) \in A, s \in(t, T), \\ \dot{Y}(s)=\frac{1}{\varepsilon} g_{i}(X(s), Y(s), \alpha(s)) & \text { for } Y(s) \in \Omega_{i}, i=1,2, \alpha(s) \in A, s \in(t, T), \\ (X(t), Y(t))=(x, y), & \end{cases}
$$

where $\varepsilon>0, A$ is compact, $f$ and $g_{i}$ are Lipschitz continuous in the state variables and continuous. The optimal control problem that we are interested in is of Mayer's type:

$$
v^{\varepsilon}(t, x, y):=\inf _{\alpha(\cdot)}\{\varphi(X(T), Y(T))\}
$$

where $\varphi$ is Lipschitz continuous.

The goal of this paper is to obtain a characterization of the limit of $v^{\varepsilon}$ as $\varepsilon$ goes to zero. Singular perturbation problems for deterministic controlled systems have been studied by many authors; see e.g., the books by Kokotović, Khalil, and O'Reilly [17], and Bensoussan [6], as well as the articles by Gaitsgory [15], Bagagiolo and Bardi [7], Alvarez and Bardi [1, 2], and the references therein.

${ }^{*}$ This work was partially supported by the project European Union under the 7th Framework Programme FP7-PEOPLE-2010-ITN, GA number 264735-SADCO, and the project ANR-HJnet: ANR-12-BS01-0008-01.

†Ceremade, Université Paris-Dauphine, Place du Maréchal de Lattre de Tassigny, F-75775 Paris Cedex 16, France (forcadel@ceremade.dauphine.fr).

${ }^{\ddagger}$ ENSTA ParisTech \& INRIA-Saclay, 828, Boulevard des Maréchaux, 91762 Palaiseau Cedex, France (Zhiping.Rao@ensta-paristech.fr). 
However, up to our knowledge, there is no result for this kind of problem on multi-domains. In our setting, the dynamics of the fast state variable $Y(\cdot)$ switch to $g_{i}$ when $Y(\cdot)$ goes into $\Omega_{i}$. Then the definitions for the dynamical system (1.1) and the optimal control problem are not clear since the dynamics of $Y(\cdot)$ is not continuous on $\mathbb{R}^{2}$. The subject of optimal control problems on multi-domains is quite recent and we would like to refer to $[4,8,10,16,20,21]$. The main difficulty lies in finding out the proper junction condition between $\Omega_{1}$ and $\Omega_{2}$ to characterize the value function of optimal control problems. Thanks to the recent work [10] on optimal control problems on stratified domains and [21] on the HJB equations on multi-domains, optimal control problems on multi-domains can be associated to HJB equations with discontinuity by introducing the concept of Essential Hamiltonians. The existence and uniqueness result for the solution of HJB equations with essential Hamiltonians has been established in [21]. Roughly speaking, the idea of this essential Hamiltonians consists in selecting the useful dynamics on the interfaces between $\Omega_{1}$ and $\Omega_{2}$ that drive the trajectories either to go into the interior of $\Omega_{i}$ or to travel on the interfaces between them. The value function $v^{\varepsilon}$ is then characterized as the unique solution of

$$
-\partial_{t} v^{\varepsilon}(t, x, y)+H^{E}\left(x, y, D_{x} v^{\varepsilon}(t, x, y), \frac{1}{\varepsilon} D_{y} v^{\varepsilon}(t, x, y)\right)=0 \text { on }(0, T) \times \mathbb{R}^{d} \times \mathbb{R}^{2},
$$

where $H^{E}$ is the essential Hamiltonian (see Definition 2.1 below), with the final condition

$$
v^{\varepsilon}(T, x, y)=\varphi(x, y) \text { on } \mathbb{R}^{d} \times \mathbb{R}^{2} .
$$

We are interested in the limit behavior as $\varepsilon \rightarrow 0$ of the solution for the above HJB equation. However, this essential Hamiltonian $H^{E}$ is not necessarily Lipschitz continuous, which is a significant difficulty. There are some works $[5,19]$ dealing with the homogenization of metric Hamilton-Jacobi equations where the Hamiltonians are continuous and coercive. But when the Hamiltonians become discontinuous, this problem remains a difficult issue. In [19], an algorithm has been introduced to solve the piecewise-periodic problems numerically where the Hamiltonians are not continuous, but there is no general theoretical result for this method.

In this paper, we consider coercive Hamiltonians by assuming a controllability condition on the fast variable $Y(\cdot): \exists r_{0}>0$,

$$
B_{\mathbb{R}^{2}}\left(0, r_{0}\right) \subseteq\left\{g_{i}(x, y, a), a \in A\right\}, \forall x \in \mathbb{R}^{d}, y \in \mathbb{R}^{2}, i=1,2 .
$$

We also assume that the multi-domains have a periodic structure so that the dynamics for $Y(\cdot)$ is bounded. Our main result states that the limit $v(t, x)$, as $\varepsilon \rightarrow 0$, of the value function $v^{\varepsilon}(t, x, y)$ is the unique solution of

$$
-\partial_{t} v(t, x)+\bar{H}\left(x, D_{x} v(t, x)\right)=0 \text { on }(0, T) \times \mathbb{R}^{d} \text {, and } v(T, x)=\inf _{y \in \mathbb{R}^{2}} \varphi(x, y) \text { on } \mathbb{R}^{d} \text {. }
$$

The Hamiltonian $\bar{H}$ is called the effective Hamiltonian and is classically determined by the following cell problem: for each fixed $x \in \mathbb{R}^{d}, P \in \mathbb{R}^{d}$, there exists a unique constant $\bar{H}(x, P)$ such that the cell problem

$$
H^{E}\left(x, y, P, D_{y} w(y)\right)=\bar{H}(x, P)
$$

has a periodic viscosity solution $w$. 
To solve the cell problem, we classically introduce an approximated cell problem (see $[18,13])$. However, the essential Hamiltonian $H^{E}$ which appears in this approximating cell problem is not continuous. Thus, the construction of approximated corrector is a difficult issue. To solve this problem, we use the fact that the essential Hamiltonian is defined from an optimal control point of view and we show that approximated correctors can be constructed as the value functions of infinite horizon optimal control problems.

Another difficulty is to prove that approximated correctors converge toward a corrector of the cell problem. This uses a stability result which we prove in the framework of discontinuous hamiltonian (but only for Lipschitz continuous solutions).

1.1 Setting of the problem We are interested in the limit value of the optimal control problems of Mayer's type. Let $T>0$ be a fixed final time and $\mathcal{A}$ be the set of controls given by

$$
\mathcal{A}:=\left\{\alpha:(0, T) \rightarrow \mathbb{R}^{m} \text { measurable functions, } \alpha(t) \in A \text { a.e. in }(0, T)\right\}
$$

with $A$ being a compact subset of $\mathbb{R}^{m}$. In the sequel, all the periodic functions we consider have the period

$$
S=(-1,1)^{2}
$$

then " $f$ is $S$-periodic" means:

$$
\forall k \in \mathbb{Z}^{2}, \forall x \in \mathbb{R}^{2}, f(x+2 k)=f(x) .
$$

We assume that the function $f: \mathbb{R}^{d} \times \mathbb{R}^{2} \times A \rightarrow \mathbb{R}^{d}$ satisfies the following:

(H1) $\left\{\begin{array}{l}\text { (i) } \quad \forall x \in \mathbb{R}^{d}, y \in \mathbb{R}^{2},\{f(x, y, a): a \in A\} \text { is nonempty, convex, and compact; } \\ \text { (ii) } f(x, y, a) \text { is } L \text {-Lipschitz continuous w.r.t } x, y \text {, and continuous w.r.t } a \text {; } \\ \text { (iii) } \exists M>0 \text { so that }\|f(x, y, a)\| \leq M, \forall,(x, y) \in \mathbb{R}^{d} \times \mathbb{R}^{2}, a \in A \text {. }\end{array}\right.$

For $i=1,2$, we assume that the functions $g_{i}: \mathbb{R}^{d} \times \mathbb{R}^{2} \times A \rightarrow \mathbb{R}^{2}$ satisfies the following assumption

(H2) $\left\{\begin{array}{l}\text { (i) } \forall x \in \mathbb{R}^{d}, y \in \mathbb{R}^{2},\left\{g_{i}(x, y, a): a \in A\right\} \text { is nonempty, convex, and compact; } \\ \text { (ii) } g_{i}(x, y, a) \text { is } L \text {-Lipschitz continuous w.r.t } x, y \text {, and continuous w.r.t } a ; \\ \text { (iii) } \exists r_{0}>0 \text { so that } \forall(x, y) \in \mathbb{R}^{d} \times \mathbb{R}^{2}, B\left(0, r_{0}\right) \subseteq\left\{g_{i}(x, y, a): a \in A\right\} ; \\ \text { (iv) } \forall x \in \mathbb{R}^{d}, a \in A, g_{i}(x, \cdot, a) \text { is } S \text {-periodic. }\end{array}\right.$

We consider the following periodic chessboard structure (see also Figure 1.1)

$$
\begin{aligned}
& S_{1}:=\left\{(0,1) \times(0,1)+k S, k \in \mathbb{Z}^{2}\right\} \cup\left\{(-1,0) \times(-1,0)+k S, k \in \mathbb{Z}^{2}\right\}, \Omega_{1}:=\bigcup_{\mathcal{M} \in S_{1}} \mathcal{M}, \\
& S_{2}:=\left\{(-1,0) \times(0,1)+k S, k \in \mathbb{Z}^{2}\right\} \cup\left\{(0,1) \times(-1,0)+k S, k \in \mathbb{Z}^{2}\right\} . \Omega_{2}:=\bigcup_{\mathcal{M} \in S_{2}} \mathcal{M} .
\end{aligned}
$$




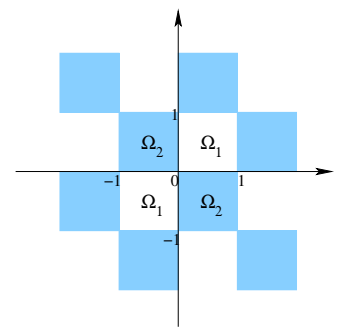

FIG. 1.1. The periodic chessboard structure.

Remark 1.1. The structure of multi-domains we considered here is the type of chessboard structure. In fact, due to the work [10, 21] our results can be generalized on any periodic structure of multi-domains $\left(\mathcal{M}_{i}\right)_{i=1, \ldots, n}, n \in \mathbb{N}$ satisfying the following: each $\mathcal{M}_{i}$ is a $C^{2}$ open embedded 2-manifold in $\mathbb{R}^{2}$, each $\overline{\mathcal{M}}_{i}$ is proximally smooth and wedged, and

$$
S=\bigcup_{i=1}^{n} \overline{\mathcal{M}}_{i}, \mathcal{M}_{i} \cap \mathcal{M}_{j}=\emptyset \text { for } i \neq j, i, j=1, \ldots, n .
$$

The concepts of proximally smooth and wedged are introduced in [12]. For any set $\mathcal{M} \subseteq \mathbb{R}^{d}$, we recall that $\overline{\mathcal{M}}$ is proximally smooth means that the signed distance function to $\overline{\mathcal{M}}$ is differentiable on a tubular neighborhood of $\overline{\mathcal{M}}$. $\overline{\mathcal{M}}$ is said to be wedged means that the interior of $\mathcal{T}_{\overline{\mathcal{M}}}(x)$ is nonempty for each $x \in \overline{\mathcal{M}}$. Here $\mathcal{T}_{\overline{\mathcal{M}}}(x)$ is the tangent cone of $\overline{\mathcal{M}}$ at $x$ defined by

$$
\mathcal{T}_{\overline{\mathcal{M}}}(x)=\left\{\zeta \in \mathbb{R}^{2}: \liminf _{t \rightarrow 0^{+}} \frac{d_{\overline{\mathcal{M}}}(x+t \zeta)}{t}=0\right\},
$$

where $d_{\overline{\mathcal{M}}}(\cdot)$ is the distance function to $\overline{\mathcal{M}}$.

Now in order to well define a dynamical system on the whole $\mathbb{R}^{2}$ for $Y(\cdot)$, we need to determine the dynamics on the interfaces between the sets of $S_{1}$ and $S_{2}$. The idea is to consider the approach of Filippov regularization of the dynamics around the interfaces, i.e. consider the multifunction $\Phi: \mathbb{R}^{d} \times \mathbb{R}^{2} \rightsquigarrow \mathbb{R}^{2}$ defined by

$$
\Phi(x, y):= \begin{cases}\Phi_{i}(x, y) & \text { if } y \in \Omega_{i} \\ \overline{c o}\left(\Phi_{1}(x, y), \Phi_{2}(x, y)\right) & \text { otherwise }\end{cases}
$$

where

$$
\Phi_{i}(x, y):=\left\{\left(\begin{array}{c}
f(x, y, a) \\
g_{i}(x, y, a)
\end{array}\right), a \in A\right\}, \forall(x, y) \in \mathbb{R}^{d} \times \mathbb{R}^{2}, i=1,2,
$$

and $\overline{c o}\left(\Phi_{1}(x, y), \Phi_{2}(x, y)\right)$ is defined as the set

$$
\left\{(1-\theta)\left(\begin{array}{c}
f\left(x, y, a_{1}\right) \\
g_{1}\left(x, y, a_{1}\right)
\end{array}\right)+\theta\left(\begin{array}{c}
f\left(x, y, a_{2}\right) \\
g_{2}\left(x, y, a_{2}\right)
\end{array}\right) \mid \theta \in[0,1], a_{1}, a_{2} \in A\right\} .
$$

Now we are ready to introduce the optimal control problem. Given the initial time $t \in[0, T]$ and the initial state $(x, y) \in \mathbb{R}^{d} \times \mathbb{R}^{2}$, we consider the controlled trajectories $(X, Y)(\cdot):[0, T] \rightarrow \mathbb{R}^{d} \times \mathbb{R}^{2}$ satisfying

$$
\left\{\begin{array}{c}
\left(\begin{array}{c}
\dot{X}(s) \\
\varepsilon \dot{Y}(s)
\end{array}\right) \in \Phi(X(s), Y(s)) \quad \text { for } s \in(t, T), \\
X(t)=x, Y(t)=y .
\end{array}\right.
$$


We denote by $S_{[t, T]}^{\varepsilon}(x, y)$ the set of absolutely continuous trajectories satisfying (1.2). Let $\varphi: \mathbb{R}^{d} \times \mathbb{R}^{2} \rightarrow \mathbb{R}$ be a bounded Lipschitz continuous function. Consider the following Mayer's problem: for any $\varepsilon>0$,

$$
v^{\varepsilon}(t, x, y):=\inf \left\{\varphi(X(T), Y(T)):(X(\cdot), Y(\cdot)) \in S_{[t, T]}^{\varepsilon}(x, y)\right\} .
$$

Note that $\Phi$ is upper semi-continuous and convex valued, but $\Phi$ is not necessarily Lipschitz continuous. The characterization of the value function via the HamiltonJacobi-Bellman approach is a difficult issue and we refer to [21] in order to prove that $v^{\varepsilon}$ is the unique solution of

(1.4)

$$
\begin{cases}-\partial_{t} v^{\varepsilon}(t, x, y)+H^{E}\left(x, y, D_{x} v^{\varepsilon}(t, x, y), \frac{1}{\varepsilon} D_{y} v^{\varepsilon}(t, x, y)\right)=0 & \text { on }(0, T) \times \mathbb{R}^{d} \times \mathbb{R}^{2} \\ v^{\varepsilon}(T, x, y)=\varphi(x, y) & \text { on } \mathbb{R}^{d} \times \mathbb{R}^{2}\end{cases}
$$

where $H^{E}$ is the essential Hamiltonian which is discontinuous in general and will be defined in Section 2.

1.2 Main results We now want to characterize the limit $v$ of $v^{\varepsilon}$ as the velocity of the fast variable goes to infinity (i.e. $\varepsilon \rightarrow 0$ ).

The main results are the following.

Theorem 1.2 (Definition of the effective Hamiltonian). For each fixed $x \in \mathbb{R}^{d}, P \in$ $\mathbb{R}^{d}$, there exists a unique $\lambda:=\bar{H}(x, P) \in \mathbb{R}$ such that the cell problem

$$
H^{E}\left(x, y, P, D_{y} w(y)\right)=\lambda
$$

has a periodic viscosity solution $w$. Moreover, seen as a function of $x$ and $P, \bar{H}$ is Lipschitz continuous.

Theorem 1.3 (Convergence result). Assume (H1)-(H2). The value function $v^{\varepsilon}$ defined in (1.3) converges uniformly on $[0, T] \times \mathbb{R}^{d} \times \mathbb{R}^{2}$ to the unique viscosity solution $v$ of

$$
\begin{cases}-\partial_{t} v(t, x)+\bar{H}\left(x, D_{x} v(t, x)\right)=0 & \text { for } t \in(0, T), x \in \mathbb{R}^{d} \\ v(T, x)=\inf _{y \in \mathbb{R}^{2}} \varphi(x, y) & \text { for } x \in \mathbb{R}^{d}\end{cases}
$$

Note the fact that the limiting equation does not depend on the fast variable, (1.6) can be understood by looking at the controllability assumptions which implies that at the limit, the fast variable can travel over all the space $\mathbb{R}^{2}$ with infinite velocity (this also explains the terminal condition).

We also want to point out that the effective Hamiltonian $\bar{H}$ is Lipschitz continuous in $x$ and so the perturbed test function (introduced by Evans [13]) can be adapted to our case.

The paper is organized as follows. In section 2, we give some preliminary results including the notion of essential Hamiltonians. Section 3 discusses the cell problem while Section 4 is devoted to the properties of the effective Hamiltonian $\bar{H}$. The proof of the convergence result is given in Section 5 .

2 Preliminary results We now state the definition of the essential Hamiltonian. Note that we have two types of interfaces according to their dimensions, we set

$$
I:=\left\{(k, k+1) \times\{m\},(k, m) \in \mathbb{Z}^{2}\right\} \cup\left\{\{k\} \times(m, m+1),(k, m) \in \mathbb{Z}^{2}\right\} \cup \mathbb{Z}^{2}
$$


as the union of all the 1-dimensional interfaces and 0-dimensional interfaces.

For any $\mathcal{M} \in S_{1} \cup S_{2} \cup I$, we denote by $\Phi_{\mathcal{M}}: \mathbb{R}^{d} \times \mathbb{R}^{2} \rightsquigarrow \mathbb{R}^{d} \times \mathbb{R}^{2}$ defined by

$$
\Phi_{\mathcal{M}}(x, y):= \begin{cases}\Phi_{i}(x, y) & \text { if } \mathcal{M} \in S_{i}, i=1,2, \\ \Phi(x, y) & \text { if } \mathcal{M} \in I .\end{cases}
$$

Consider the essential multifunction $\Phi^{E}$ (introduced in [10, 21]) defined as follows.

Definition 2.1. [Essential dynamics and essential Hamiltonian] Let $\Phi^{E}: \mathbb{R}^{d} \times \mathbb{R}^{2} \rightsquigarrow$ $\mathbb{R}^{d} \times \mathbb{R}^{2}$ be a multifunction defined for any $(x, y) \in \mathbb{R}^{d} \times \mathbb{R}^{2}$ by

$$
\Phi^{E}(x, y):=\bigcup_{\mathcal{M} \in S_{1} \cup S_{2} \cup I, y \in \overline{\mathcal{M}}}\left(\Phi_{\mathcal{M}}(x, y) \cap\left(\mathbb{R}^{d} \times \mathcal{T}_{\overline{\mathcal{M}}}(y)\right)\right) .
$$

We also denote by $H^{E}: \mathbb{R} \times \mathbb{R} \rightarrow \mathbb{R}$ the essential Hamiltonian defined by

$$
H^{E}(x, y, \xi, \zeta):=\sup _{(p, q) \in \Phi^{E}(x, y)}\{-p \cdot \xi-q \cdot \zeta\} .
$$

Example 1. Here we give a precise example to see more clearly the elements in $\Phi^{E}$. We ignore the variable $X$ since there is no singularity in the structure of the dynamics of $X$. Consider $g_{1} \equiv(1,1)$ and $g_{2} \equiv(-1,1)$, Figure 2.1 shows the differences between $\Phi$ and $\Phi^{E}$ on the interfaces (elements in I. In fact, on the interfaces $\Phi$ contains

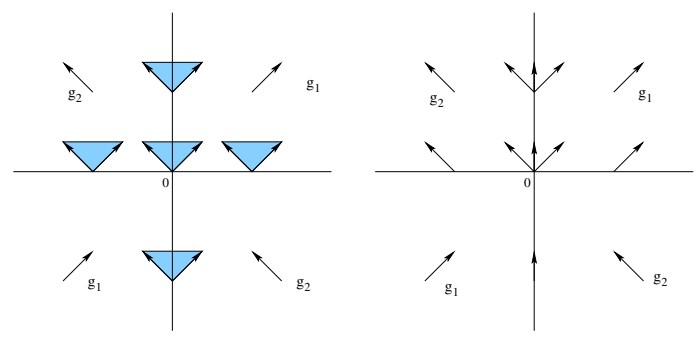

FIG. 2.1. $\Phi$ and $\Phi^{E}$.

all the possible directions (the whole triangles) in which some of them may be useless. While the definition of $\Phi^{E}$ allows to select only the useful dynamics for the trajectories in $S_{[t, T]}^{\varepsilon}(x, y)$ : the directions $g_{i}$ which are inward for $\bar{\Omega}_{i}$ and the tangent directions for the interfaces. We refer to [10, 21] for more details.

Remark 2.2. $\Phi^{E}(x, y)$ is Lipschitz continuous in $x$ since $\Phi(\cdot, y)$ is Lipschitz continuous. However, $\Phi^{E}(x, y)$ is not necessarily continuous in $y$ because of the geometrical singularity of the dynamical structure for the variable $y$. Therefore, the essential Hamiltonian $H^{E}(x, y, \xi, \zeta)$ is Lipschitz continuous in $x$, but not necessarily continuous in $y$.

Then here is the characterization result ([21, Theorem 2.4]) for the value function. Lemma 2.3 (Characterization of the value function). The value function $v^{\varepsilon}$ is unique Lipschitz continuous viscosity solution of (1.4) in the sense of Definition 2.5.

Before giving the definition of viscosity solution, we need the following notion of extended differentials.

Definition 2.4 (Extended differential). Let $\phi:(0, T) \times \mathbb{R}^{d} \times \mathbb{R}^{2} \rightarrow \mathbb{R}$ be a continuous function and $\mathcal{M} \in S_{1} \cup S_{2} \cup I$. Suppose that $\phi \in C^{1}\left((0, T) \times \mathbb{R}^{d} \times \overline{\mathcal{M}}\right)$, then for any $t \in(0, T), x \in \mathbb{R}^{d}, y \in \overline{\mathcal{M}}$, the extended differential of $\phi$ on $(t, x, y)$ is defined by

$$
D_{\overline{\mathcal{M}}} \phi(t, x, y):=\lim _{z \rightarrow y, z \in \mathcal{M}} D \phi(t, x, z) .
$$


Note that since $D \phi(t, x, \cdot)$ is continuous on $\overline{\mathcal{M}}$, the extended differential is nothing but the extension of $D \phi(t, x, \cdot)$ to the whole $\overline{\mathcal{M}}$.

We now state the definition of viscosity solution for (1.4).

Definition 2.5 (Viscosity solution for (1.4)). Let $u:(0, T] \times \mathbb{R}^{d} \times \mathbb{R}^{2} \rightarrow \mathbb{R}$ be a bounded Lipschitz continuous function.

(i) We say that $u$ is a supersolution of (1.4) if for any $\left(t_{0}, x_{0}, y_{0}\right) \in(0, T) \times \mathbb{R}^{d} \times \mathbb{R}^{2}$, $\phi \in C^{1}\left((0, T) \times \mathbb{R}^{d} \times \mathbb{R}^{2}\right)$ such that $u-\phi$ attains a local minimum on $\left(t_{0}, x_{0}, y_{0}\right)$, we have

$$
-\phi_{t}\left(t_{0}, x_{0}, y_{0}\right)+H^{E}\left(x_{0}, y_{0}, D_{x} \phi\left(t_{0}, x_{0}, y_{0}\right), \frac{1}{\varepsilon} D_{y} \phi\left(t_{0}, x_{0}, y_{0}\right)\right) \geq 0 .
$$

(ii) We say that $u$ is a subsolution of (1.4) if for any $\left(t_{0}, x_{0}, y_{0}\right) \in(0, T) \times \mathbb{R}^{d} \times \mathbb{R}^{2}$,

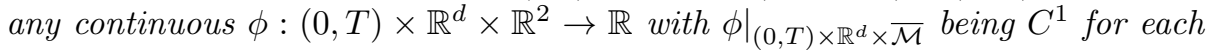
$\mathcal{M} \in S_{1} \cup S_{2} \cup I$ with $y_{0} \in \overline{\mathcal{M}}$ such that $u-\phi$ attains a local maximum at $\left(t_{0}, x_{0}, y_{0}\right)$, we have

$$
\begin{aligned}
-\phi_{t}\left(t_{0}, x_{0}, y_{0}\right)+\sup _{(p, q) \in \Phi_{\mathcal{M}}\left(x_{0}, y_{0}\right) \cap\left(\mathbb{R}^{d} \times \mathcal{T}_{\overline{\mathcal{M}}}\left(y_{0}\right)\right)}\{ & -p \cdot D_{x} \phi\left(t_{0}, x_{0}, y_{0}\right) \\
& \left.-\frac{1}{\varepsilon} q \cdot D_{\overline{\mathcal{M}}} \phi\left(t_{0}, x_{0}, y_{0}\right)\right\} \leq 0 .
\end{aligned}
$$

(iii) We say that $u$ is a viscosity solution of (1.4) if $u$ is both a supersolution and a subsolution, and $u$ satisfies the final condition

$$
u(T, x, y)=\varphi(x, y), \forall(x, y) \in \mathbb{R}^{d} \times \mathbb{R}^{2} .
$$

In the following, we will also use different equations (in particular for the cell problem and for the approximated cell problem). We then give the definition of viscosity solution for a more general equation of the form

$$
H_{1}(u(y))+H^{E}(x, y, P, D u(y))=0 .
$$

Definition 2.6 (Viscosity solution for (2.1)). Let $u: \mathbb{R}^{2} \rightarrow \mathbb{R}$ be a bounded Lipschitz continuous function.

(i) We say that $u$ is a supersolution of (2.1) if for any $y_{0} \in \times \mathbb{R}^{2}, \phi \in C^{1}\left(\mathbb{R}^{2}\right)$ such that $u-\phi$ attains a local minimum on $y_{0}$, we have

$$
H_{1}\left(u\left(y_{0}\right)\right)+H^{E}\left(x, y_{0}, P, D \phi\left(y_{0}\right)\right) \geq 0 .
$$

(ii) We say that $u$ is a subsolution o (2.1) if for any $y_{0} \in \mathbb{R}^{2}$, any continuous $\phi: \mathbb{R}^{2} \rightarrow \mathbb{R}$ with $\left.\phi\right|_{\overline{\mathcal{M}}}$ being $C^{1}$ for each $\mathcal{M} \in S_{1} \cup S_{2} \cup I$ with $y_{0} \in \overline{\mathcal{M}}$ such that $u-\phi$ attains a local maximum at $y_{0}$, we have

$$
\left.H_{1}\left(u\left(y_{0}\right)\right)+\sup _{(p, q) \in \Phi_{\mathcal{M}}\left(x, y_{0}\right) \cap\left(\mathbb{R}^{d} \times \mathcal{T}_{\overline{\mathcal{M}}}\left(y_{0}\right)\right)}\left\{-p \cdot P-q \cdot D_{\overline{\mathcal{M}}} \phi\left(y_{0}\right)\right)\right\} \leq 0 .
$$

(iii) We say that $u$ is a viscosity solution of (2.1) if $u$ is both a supersolution and a subsolution.

We now state a comparison principle for the equation (1.4) on bounded domain Theorem 2.7 (Comparison principle in bounded domain). For any open bounded $\Omega \subseteq(0, T) \times \mathbb{R}^{d}$, let $u_{1}, u_{2}:(0, T) \times \mathbb{R}^{d} \times \mathbb{R}^{2} \rightarrow \mathbb{R}$ be Lipschitz continuous. If $u_{1}$ is a subsolution of (1.4) and $u_{2}$ is a supersolution of (1.4) on $\Omega \times \mathbb{R}^{2}$, then we have

$$
\max _{(t, x, y) \in \Omega \times \mathbb{R}^{2}}\left\{u_{1}(t, x, y)-u_{2}(t, x, y)\right\} \leq \max _{(t, x, y) \in \partial \Omega \times \mathbb{R}^{2}}\left\{u_{1}(t, x, y)-u_{2}(t, x, y)\right\} .
$$


Before we start the proof, we have the following lemma which is direct consequence of [21, Theorem 3.7, Theorem 3.11].

Lemma 2.8 (Dynamics programming principle). Let $u:(0, T) \times \mathbb{R}^{d} \times \mathbb{R}^{2}$ be Lipschitz continuous.

- If $u$ is a supersolution of (1.4), then for any $(t, x, y) \in[0, T] \times \mathbb{R}^{d} \times \mathbb{R}^{2}$ there exists $(\bar{X}, \bar{Y}) \in S_{[t, T]}^{\varepsilon}(x, y)$ such that

$$
u(t, x, y) \geq u(t+h, \bar{X}(t+h), \bar{Y}(t+h)), \text { for } 0 \leq h \leq T-t
$$

- If $u$ is a subsolution of (1.4), then for any $(t, x, y) \in[0, T] \times \mathbb{R}^{d} \times \mathbb{R}^{2}$ and any $(X, Y) \in S_{[t, T]}^{\varepsilon}(x, y)$

$$
u(t, x, y) \leq u(t+h, X(t+h), Y(t+h)), \text { for } 0 \leq h \leq T-t
$$

Proof. [Proof of Theorem 2.7] For any $\left(t_{0}, x_{0}, y_{0}\right) \in \Omega \times \mathbb{R}^{2}, u_{2}$ is a supersolution on $\Omega$ implies that there exists an absolutely continuous function $(\bar{X}, \bar{Y}) \in S_{\left[t_{0}, T\right]}^{\varepsilon}\left(x_{0}, y_{0}\right)$ such that

$$
u_{2}\left(t_{0}, x_{0}, y_{0}\right) \geq u_{2}\left(t_{0}+h, \bar{X}\left(t_{0}+h\right), \bar{Y}\left(t_{0}+h\right)\right), \text { for } 0 \leq h \leq h_{0}
$$

where

$$
h_{0}:=\inf \left\{h>0:\left(t_{0}+h, \bar{X}\left(t_{0}+h\right)\right) \notin \Omega\right\} .
$$

$u_{1}$ is a subsolution on $\Omega$ implies that

$$
u_{1}\left(t_{0}, x_{0}, y_{0}\right) \leq u_{1}\left(t_{0}+h, \bar{X}\left(t_{0}+h\right), \bar{Y}\left(t_{0}+h\right)\right), \text { for } 0 \leq h \leq h_{0}
$$

We then deduce that

$$
\left(u_{1}-u_{2}\right)\left(t_{0}, x_{0}, y_{0}\right) \leq\left(u_{1}-u_{2}\right)\left(t_{0}+h_{0}, \bar{X}\left(t_{0}+h\right), \bar{Y}\left(t_{0}+h\right)\right) .
$$

The definition of $h_{0}$ implies that $\left(t_{0}+h_{0}, \bar{X}\left(t_{0}+h\right)\right) \in \partial \Omega$, then we obtain

$$
u_{1}\left(t_{0}, x_{0}, y_{0}\right)-u_{2}\left(t_{0}, x_{0}, y_{0}\right) \leq \max _{(t, x, y) \in \partial \Omega \times \mathbb{R}^{2}}\left\{u_{1}(t, x, y)-u_{2}(t, x, y)\right\}
$$

which leads to the desired result.

3 The cell problem In this section, we focus on the the cell problem: given $x \in \mathbb{R}^{d}, P \in \mathbb{R}^{d}$, find $\lambda \in \mathbb{R}$ such that the equation (1.5) has a viscosity solution.

3.1 Approximating problem To solve the cell problem, we classically introduce an approximated cell problem. Given $x \in \mathbb{R}^{d}, P \in \mathbb{R}^{d}$ and $\beta>0$, we consider the problem

$$
\beta v^{\beta}(y)+H^{E}\left(x, y, P, D v^{\beta}(y)\right)=0, y \in \mathbb{R}^{2} .
$$

Then we investigate the limit of the approximating equation (3.1) as $\beta \rightarrow 0$ by proving that $v^{\beta} \rightarrow v$ and $\beta v^{\beta} \rightarrow-\lambda$ with $v$ solution of $(1.5)$

Since $H^{E}$ is not Lipschitz continuous in $y$, the existence and uniqueness of the solution for (3.1) need to be carrefully studied. A simple idea is to link the HJB 
equation (3.1) with an optimal control problem. For any $y \in \mathbb{R}^{2}$, we denote the set of absolutely continuous trajectories by

$$
S[x, y]:=\{(X, Y),(\dot{X}(s), \dot{Y}(s)) \in \Phi(x, Y(s)), X(0)=x, Y(0)=y\} .
$$

Given $P \in \mathbb{R}^{2}$, consider the value function $w^{\beta}$ of the following infinite horizon optimal control problem:

$$
w^{\beta}(y):=\min _{(X, Y) \in S[x, y]} \int_{0}^{+\infty} e^{-\beta s} P \cdot \dot{X}(s) d s .
$$

The main result of this subsection is the following characterization of the value function $w^{\beta}$ :

Theorem 3.1 (Characterization of the value function $w^{\beta}$ ). The value function $w^{\beta}$ is the unique viscosity solution of (3.1) in the sense of Definition 2.6.

We begin by the existence part. As in the classical case (see $[9$, Proposition III.2.5]), $w^{\beta}$ satisfies a Dynamical programming principle (DPP).

Proposition 3.2 (Dynamic programming principle). Assume that (H1) hold. Then for any $y \in \mathbb{R}^{2}, h \geq 0$, the following holds.

(i) The super-optimality. $\exists(\bar{X}, \bar{Y}) \in S[x, y]$ such that

$$
w^{\beta}(y) \geq \int_{0}^{h} e^{-\beta s} P \cdot \dot{\bar{X}}(s) d s+e^{-\beta h} w^{\beta}(\bar{Y}(h))
$$

(ii) The sub-optimality. $\forall(X, Y) \in S[x, y]$ we have

$$
w^{\beta}(y) \leq \int_{0}^{h} e^{-\beta s} P \cdot \dot{X}(s) d s+e^{-\beta h} w^{\beta}(Y(h)) .
$$

The value function $w^{\beta}$ satisfies the following properties.

Proposition 3.3 (Regularity of $\left.w^{\beta}\right)$. Assume that $(\mathbf{H 1})-(\mathbf{H 2})$ hold. Then $w^{\beta}$ is bounded and Lipschitz continuous. Moreover, the Lipschitz constant is uniform in $\beta$.

Proof. By the definition of $w^{\beta}$, for any $y \in \mathbb{R}^{2}$,

$$
\left|w^{\beta}(y)\right| \leq \int_{0}^{+\infty} e^{-\beta s}\|P\| M d s=\frac{\|P\| M}{\beta} .
$$

Now we prove the Lipschitz continuity. For any $y, z \in \mathbb{R}^{2}$, consider the following trajectory:

$$
Y(s):=y+r_{0} \frac{z-y}{\|y-z\|} s, \text { for } s \geq 0 .
$$

We set $h=\|y-z\| / r_{0}$, then we have $Y(0)=y, Y(h)=z$. Note that $\left\|\dot{y}_{x}(s)\right\|=r_{0}$, by (H2)(iii) there exists $X$ such that $(X, Y) \in S[x, y]$. Since $w^{\beta}$ satisfies the sub- 
optimality along $(X, Y)$, we obtain

$$
\begin{aligned}
w^{\beta}(y) & \leq \int_{0}^{h} e^{-\beta s} P \cdot \dot{X}(s) d s+e^{-\beta h} w^{\beta}(z) \\
& \leq w^{\beta}(z)+\int_{0}^{h} e^{-\beta s} P \cdot \dot{X}(s) d s+\left(e^{-\beta h}-1\right) w^{\beta}(z) \\
& \leq w^{\beta}(z)+\int_{0}^{h} e^{-\beta s}\|P\| M d s+\left(1-e^{-\beta h}\right)\left|w^{\beta}(z)\right| \\
& \leq w^{\beta}(z)+2\left(1-e^{-\beta h}\right) \frac{\|P\| M}{\beta} \\
& \leq w^{\beta}(z)+2 h\|P\| M=w^{\beta}(z)+\frac{2\|P\| M}{r_{0}}\|y-z\|
\end{aligned}
$$

which implies the Lipschitz continuity of $w^{\beta}$ (the Lipschitz constant is independent on $\beta$ ).

Then we have that $w^{\beta}$ is solution of the equation (3.1).

Proposition $3.4\left(w^{\beta}\right.$ satisfies (3.1)). The value function $w^{\beta}$ is the viscosity solution of $(3.1)$.

Proof. We first prove that $w^{\beta}$ is a supersolution. For any $y_{0} \in \mathbb{R}^{2}$, let $\phi \in C^{1}\left(\mathbb{R}^{2}\right)$ such that $u-\phi$ attains a local minimum on $y_{0}$. By the super-optimality satisfied by $w^{\beta}, \exists(\bar{X}, \bar{Y}) \in S\left[y_{0}\right]$ such that

$$
w^{\beta}\left(y_{0}\right) \geq \int_{0}^{h} e^{-\beta s} P \cdot \dot{\bar{X}}(s) d s+e^{-\beta h} w^{\beta}(\bar{Y}(h)) .
$$

By definition of $\phi$, we have

$$
w^{\beta}\left(y_{0}\right)-\phi\left(y_{0}\right) \leq w^{\beta}(\bar{Y}(h))-\phi(\bar{Y}(h)), \forall h>0 .
$$

Then, (3.3) and (3.4) imply that

$$
w^{\beta}\left(y_{0}\right) \geq \int_{0}^{h} e^{-\beta s} P \cdot \dot{\bar{X}}(s) d s+e^{-\beta h}\left(w^{\beta}\left(y_{0}\right)+\phi(\bar{Y}(h))-\phi\left(y_{0}\right)\right),
$$

i.e.

$$
\frac{1-e^{-\beta h}}{h} w^{\beta}\left(y_{0}\right)-\frac{1}{h} \int_{0}^{h} e^{-\beta s} P \cdot \dot{\bar{X}}(s) d s-\frac{e^{-\beta h}}{h} \int_{0}^{h} D \phi(\bar{Y}(s)) \cdot \dot{\bar{Y}}(s) d s \geq 0 .
$$

By [21, Lemma 3.6], there exists $h_{n} \rightarrow 0$ such that $\frac{\left(\bar{X}\left(h_{n}\right), \bar{Y}\left(h_{n}\right)\right)-\left(x, y_{0}\right)}{h_{n}} \rightarrow\left(p_{0}, q_{0}\right)$ for some $\left(p_{0}, q_{0}\right) \in \operatorname{co} \Phi^{E}\left(x, y_{0}\right)$. We then get

$$
\beta w^{\beta}\left(y_{0}\right)-p_{0} \cdot P-q_{0} \cdot D \phi\left(y_{0}\right) \geq 0
$$

which leads to

$$
\beta w^{\beta}\left(y_{0}\right)+\sup _{(p, q) \in \operatorname{co\Phi }^{E}\left(x, y_{0}\right)}\left\{-p \cdot P-q \cdot D \phi\left(y_{0}\right)\right\} \geq 0 .
$$

Since $(p, q) \mapsto-p \cdot P-q \cdot D \phi\left(y_{0}\right)$ is linear, we have

$$
\sup _{(p, q) \in \operatorname{co\Phi }^{E}\left(x, y_{0}\right)}\left\{-p \cdot P-q \cdot D \phi\left(y_{0}\right)\right\}=\sup _{(p, q) \in \Phi^{E}\left(x, y_{0}\right)}\left\{-p \cdot P-q \cdot D \phi\left(y_{0}\right)\right\} .
$$


Thus

$$
\beta w^{\beta}\left(y_{0}\right)+\sup _{(p, q) \in \Phi^{E}\left(x, y_{0}\right)}\left\{-p \cdot P-q \cdot D \phi\left(y_{0}\right)\right\} \geq 0,
$$

which ends the proof for the supersolution property.

Now we prove that $w^{\beta}$ is a subsolution. Let $\phi \in C\left(\mathbb{R}^{2}\right)$ such that $u-\phi$ attains a local maximum at $y_{0}$ with $\phi \in C^{1}(\overline{\mathcal{M}})$ for every $\mathcal{M} \in S_{1} \cup S_{2} \cup I$ such that $y_{0} \in \overline{\mathcal{M}}$. If $y_{0} \in \mathcal{M}$ with $\mathcal{M} \in S_{1} \cup S_{2}$, since $g_{1}$ and $g_{2}$ are Lipschitz continuous, then the proof is classical (see [9]) and we skip it. We then assume that $y_{0}$ lies in an element of $I$. For each $\mathcal{M} \in S_{1} \cup S_{2} \cup I$ with $y_{0} \in \overline{\mathcal{M}}$, any $(p, q) \in \Phi_{\mathcal{M}}\left(x, y_{0}\right) \cap\left(\mathbb{R}^{d} \times \mathcal{T}_{\overline{\mathcal{M}}}\left(y_{0}\right)\right)$, by [21, Lemma 3.9] there exists $h>0$ and a solution $(X, Y) \in S\left[x, y_{0}\right]$ which is $C^{1}$ on $[0, h]$ with $(\dot{X}(0), \dot{Y}(0))=(p, q)$ and $Y(s) \in \overline{\mathcal{M}}, \forall s \in[0, h]$. By the sub-optimality of $w^{\beta}$,

$$
w^{\beta}\left(y_{0}\right) \leq \int_{0}^{h} e^{-\beta s} P \cdot \dot{X}(s) d s+e^{-\beta h} w^{\beta}(Y(h)) .
$$

We have also

$$
w^{\beta}\left(y_{0}\right)-\phi\left(y_{0}\right) \geq w^{\beta}(Y(h))-\phi(Y(h)), \forall h>0 .
$$

By a similar argument as in the supersolution property case, we can deduce that

$$
\frac{1-e^{-\beta h}}{h} w^{\beta}\left(y_{0}\right)-\frac{1}{h} \int_{0}^{h} e^{-\beta s} P \cdot \dot{Y}(s) d s-\frac{e^{-\beta h}}{h} \int_{0}^{h} D \phi(Y(s)) \dot{Y}(s) d s \leq 0 .
$$

Taking $h \rightarrow 0$ leads to

$$
\beta w^{\beta}\left(y_{0}\right)-\left(p \cdot P+q \cdot D_{\overline{\mathcal{M}}} \phi\left(y_{0}\right)\right) \leq 0 .
$$

The point $(p, q)$ being arbitrary in $\Phi_{\mathcal{M}}\left(x, y_{0}\right) \cap\left(\mathbb{R}^{d} \times \mathcal{T}_{\overline{\mathcal{M}}}\left(y_{0}\right)\right)$, we deduce that

$$
\beta w^{\beta}\left(y_{0}\right)+\sup _{(p, q) \in \Phi_{\mathcal{M}}\left(x, y_{0}\right) \cap\left(\mathbb{R}^{d} \times \mathcal{T}_{\overline{\mathcal{M}}}\left(x, y_{0}\right)\right)}\left\{-p \cdot P-q \cdot D_{\overline{\mathcal{M}}} \phi\left(x_{0}\right)\right\} \leq 0,
$$

which ends the proof.

Before we prove the uniqueness result, we state the following results dealing with the relation between supersolution (resp. subsolution) and super-optimality (resp. sub-optimality).

Theorem 3.5 (Supersolution implies super-optimality). Let $u: \mathbb{R}^{2} \rightarrow \mathbb{R}$ be a supersolution of (3.1), then u satisfies the super-optimality.

Proof. We want to prove that there exists $(\bar{X}, \bar{Y}) \in S[x, y]$ such that

$$
u(y) \geq \int_{0}^{h} e^{-\beta s} P \cdot \dot{\bar{X}}(s) d s+e^{-\beta h} u(\bar{Y}(h)), \text { for } h>0,
$$

i.e.

$$
u(\bar{Y}(h)) \leq \bar{\xi}(h), \bar{\xi}(h):=e^{\beta h}\left(u(y)-\int_{0}^{h} e^{-\beta s} P \cdot \dot{\bar{X}}(s) d s\right), h>0 .
$$


For any $y \in \mathbb{R}^{2}$, consider the following viability problem:

$$
\begin{cases}(\dot{X}(h), \dot{Y}(h) \in \Phi(x, Y(h)) & \text { for } h \in(0, \infty), \\ \dot{\xi}(h)=\beta \xi(h)-P \cdot \dot{X}(h) & \text { for } h \in(0, \infty), \\ (X(0), Y(0), \xi(0))=(x, y, u(y)), & \\ (Y(h), \xi(h)) \in \text { epi }(u) . & \end{cases}
$$

For any $(y, \xi) \in$ epi $(u)$, we have $u(y) \leq \xi$. We claim that for any $(\zeta, \sigma) \in$ $\left[\mathcal{T}_{\text {epi }(u)}(y, u(y))\right]^{-*}$,

$$
\inf _{(p, q) \in \Phi(x, y)}\langle(q, \beta \xi-P \cdot p),(\zeta, \sigma)\rangle \leq 0 .
$$

Indeed, let $(\zeta, \sigma) \in\left[\mathcal{T}_{\text {epi }(u)}(y, u(y))\right]^{-}$. Since $(0,1) \in \mathcal{T}_{\text {epi }(u)}(y, u(y))$, by the definition of $\left[\mathcal{T}_{\text {epi }(u)}(y, u(y))\right]^{-}$we have

$$
\langle(\zeta, \sigma),(0,1)\rangle \leq 0,
$$

i.e. $\sigma \leq 0$. Based on this fact, we consider the following three cases.

Case 1: $\sigma=-1$

By [14, Proposition 4.1] there exists $\phi \in C^{1}\left(\mathbb{R}^{d}\right)$ such that $u-\phi$ attains a local minimum on $y$ with $D \phi(y)=\zeta$. Then

$$
\begin{aligned}
\inf _{(p, q) \in \Phi(x, y)}\langle(q, \beta \xi-P \cdot p),(\zeta,-1)\rangle & =-\beta \xi+\inf _{(p, q) \in \Phi(x, y)}\{D \phi(y) \cdot q+P \cdot p\} \\
& \leq-\beta u(y)+\inf _{(p, q) \in \Phi(x, y)}\{D \phi(y) \cdot q+P \cdot p\} \\
& \leq-\beta u(y)+\inf _{(p, q) \in \Phi^{E}(x, y)}\{D \phi(y) \cdot q+P \cdot p\} \leq 0 .
\end{aligned}
$$

Case 2 : $\sigma<0$

In that case, $(\zeta /|\sigma|,-1) \in\left[\mathcal{T}_{\text {epi }(u)}(y, u(y))\right]^{-}$. We deduce using the previous case, that

$$
\inf _{(p, q) \in \Phi(x, y)}\left\langle(q, \beta \xi-P \cdot p),\left(\frac{\zeta}{|\sigma|},-1\right)\right\rangle \leq 0,
$$

which implies

$$
\inf _{(p, q) \in \Phi(x, y)}\langle(q, \beta \xi-P \cdot p),(\zeta, \sigma)\rangle \leq 0 .
$$

Case 3 : $\sigma=0$

By [14, Lemma 4.2] there exists $y_{n} \rightarrow y,\left(\zeta_{n}, \sigma_{n}\right) \rightarrow(\zeta, 0)$ such that

$$
\left(\zeta_{n}, \sigma_{n}\right) \in\left[\mathcal{T}_{\text {epi }(u)}\left(y_{n}, u\left(y_{n}\right)\right)\right]^{-}, \sigma_{n}<0 .
$$

Using Case 2, we get that

$$
\inf _{(p, q) \in \Phi\left(x, y_{n}\right)}\left\langle(q, \beta \xi-P \cdot p),\left(\zeta_{n}, \sigma_{n}\right)\right\rangle \leq 0 .
$$

${ }^{*}\left[\mathcal{T}_{\text {epi }(u)}(y, u(y))\right]^{-}$is the negative polar cone of $\mathcal{T}_{\text {epi }(u)}(y, u(y))$, i.e. $p \in\left[\mathcal{T}_{\text {epi }(u)}(y, u(y))\right]^{-}$if and only if $\langle p, q\rangle \leq 0$ for any $q \in \mathcal{T}_{\text {epi }(u)}(y, u(y))$. 
Since $\Phi$ is upper semicontinuous, we deduce that

$$
\inf _{(p, q) \in \Phi(x, y)}\langle(q, \beta \xi-P \cdot p),(\zeta, 0)\rangle \leq 0 .
$$

which ends the proof of (3.8).

Note that

$$
\left(\begin{array}{c}
\dot{Y}(h) \\
\dot{\xi}(h)
\end{array}\right) \in\left(\begin{array}{c}
0 \\
\beta \xi(h)
\end{array}\right)+\left(\begin{array}{cc}
0 & 1 \\
-P & 0
\end{array}\right) \Phi(x, Y(h)):=\Psi(Y(h), \xi(h)),
$$

where $\Psi$ is upper semicontinuous since $\Phi$ is upper semicontinuous. Equation (3.8) can be rewritten as

$$
\inf _{(p, q) \in \Phi(x, y)}\left\langle\left(\begin{array}{c}
0 \\
\beta \xi
\end{array}\right)+\left(\begin{array}{cc}
0 & 1 \\
-P & 0
\end{array}\right)\left(\begin{array}{c}
p \\
q
\end{array}\right),\left(\begin{array}{c}
\zeta \\
\sigma
\end{array}\right)\right\rangle \leq 0
$$

which is equivalent to, by the definition of $\Psi$,

$$
\inf _{\left(p^{\prime}, q^{\prime}\right) \in \Psi(x, y)}\left\langle\left(p^{\prime}, q^{\prime}\right),(\zeta, \sigma)\right\rangle \leq 0 .
$$

Then we deduce that

$$
\Psi(y, \xi) \cap \mathcal{T}_{\text {epi }(u)}(y, u(y)) \neq \emptyset, \text { for }(y, \xi) \in \text { epi }(u) .
$$

For any $(y, \xi) \in$ epi $(u)$, if $\xi \neq u(y)$, i.e. $\xi>u(y)$, then $(y, \xi) \in$ int epi $(u)$, we have

$$
\mathcal{T}_{\text {epi }(u)}(y, \xi)=\mathbb{R}^{3} \supseteq \mathcal{T}_{\text {epi }(u)}(y, u(y)) .
$$

Thus,

$$
\Psi(y, \xi) \cap \mathcal{T}_{\text {epi }(u)}(y, \xi) \neq \emptyset, \text { for }(y, \xi) \in \text { epi }(u) .
$$

Since $(y, u(y)) \in$ epi $(u)$ and $\Psi$ are usc, the viability theorem [3, pp. 180] yields that problem $(3.7)$ has a viable solution $(\bar{X}(\cdot), \bar{Y}(\cdot), \bar{\xi}(\cdot))$, i.e.

$$
(\bar{Y}(h), \bar{\xi}(h)) \in \text { epi }(u), \forall h \geq 0,
$$

which leads to $u(\bar{Y}(h)) \leq \bar{\xi}(h), \forall h \geq 0$.

Theorem 3.6 (Subsolution implies sub-optimality). Let $u: \mathbb{R}^{2} \rightarrow \mathbb{R}$ be a subsolution of (3.1), then $u$ satisfies the sub-optimality.

To do the proof, we need the following result

Proposition 3.7. Let $u$ be a subsolution of (3.1). Suppose $\mathcal{M} \in S_{1} \cup S_{2} \cup I$ and $\Omega$ is a finite union of sets contained in $S_{1} \cup S_{2} \cup I$ with $\mathcal{M} \subseteq \bar{\Omega}$. Assume $\Omega$ has the following property: for any $0 \leq a \leq b$ and any trajectory $(X, Y) \in S[x, y]$ with $Y(\cdot) \subset \Omega$, we have

$$
u(Y(a)) \leq \int_{a}^{b} e^{-\beta(s-a)} P \cdot \dot{X}(s) d s+e^{-\beta(b-a)} u(Y(b)) .
$$

Then for any trajectory $(X, Y) \in S[x, y]$ with $Y(\cdot) \subseteq \Omega \cup \mathcal{M}$, we still have

$$
u(Y(a)) \leq \int_{a}^{b} e^{-\beta(s-a)} P \cdot \dot{X}(s) d s+e^{-\beta(b-a)} u(Y(b)) .
$$


Proof. Let $(X, Y) \in S[x, y]$ with $Y(\cdot) \subseteq \Omega \cup \mathcal{M}$ satisfying the property (3.9). Without loss of generality, suppose that $Y(a) \in \mathcal{M}, Y(b) \in \mathcal{M}$ (otherwise we consider the first arrival time and the last exit time of $Y$ for $\mathcal{M})$. Let $J:=\{s \in[a, b]: Y(s) \notin$ $\mathcal{M}$ \}, which is an open set and so can be written as

$$
J=\bigcup_{n=1}^{\infty}\left(a_{n}, b_{n}\right)
$$

where the intervals are disjoint. For a fixed $p \in \mathbb{N}$, we set

$$
J_{p}:=\bigcup_{n=1}^{p}\left(a_{n}, b_{n}\right)
$$

as the union of the first $p$ intervals which, without loss of generality, after reindexing can be assumed to satisfy

$$
a_{1}<b_{1} \leq a_{2}<b_{2} \leq \cdots \leq a_{p}<b_{p} .
$$

We set $b_{0}:=a$ and $a_{p+1}:=b$. Then $a \leq a_{1}$ and $b_{p} \leq b$. For $n=1, \ldots, p, Y(s) \in \Omega$ for $s \in\left(a_{n}, b_{n}\right)$. Let $\eta>0$ small enough such that $\left[a_{n}+\eta, b_{n}-\eta\right] \subset\left(a_{n}, b_{n}\right)$, then by $(3.9)$

$$
u\left(Y\left(a_{n}+\eta\right)\right) \leq \int_{a_{n}+\eta}^{b_{n}-\eta} e^{-\beta\left(s-a_{n}-\eta\right)} P \cdot \dot{X}(s) d s+e^{-\beta\left(b_{n}-a_{n}-2 \eta\right)} u\left(Y\left(b_{n}-\eta\right)\right) .
$$

Taking $\eta \rightarrow 0$ and by the continuity of $u, Y(\cdot)$ and the integral, we deduce that

$$
u\left(Y\left(a_{n}\right)\right) \leq \int_{a_{n}}^{b_{n}} e^{-\beta\left(s-a_{n}\right)} P \cdot \dot{X}(s) d s+e^{-\beta\left(b_{n}-a_{n}\right)} u\left(Y\left(b_{n}\right)\right) .
$$

Next we need to deal with $Y(\cdot)$ restricted to $\left[b_{n}, a_{n+1}\right]$. For $n=0, \ldots, p$, we note that $Y(s) \in \mathcal{M}$ for all $s \in\left[b_{n}, a_{n+1}\right] \backslash J$, then $(\dot{X}(s), \dot{Y}(s)) \in \Phi(x, Y(s)) \cap\left(\mathbb{R}^{d} \times \mathcal{T}_{\mathcal{M}}(Y(s))\right)$ for almost all $s \in\left[b_{n}, a_{n+1}\right] \backslash J$. For $n=0, \ldots, p$, set $\eta_{n}:=\operatorname{meas}\left(\left[b_{n}, a_{n+1}\right] \cap J\right)$, and note that $\sum_{n=0}^{p} \eta_{n}=\operatorname{meas}\left(J \backslash J_{p}\right)$. Then we have

$$
\left|\int_{b_{n}}^{a_{n+1}} e^{-\beta\left(s-b_{n}\right)} P \cdot \dot{X}(s) d s\right| \leq M|P| \eta_{n} .
$$

We now calculate how far $(X(\cdot), Y(\cdot))$ is from a trajectory lying in $\mathbb{R}^{d} \times \mathcal{M}$ with dynamics $\Phi(X(\cdot), Y(\cdot)) \cap\left(\mathbb{R}^{d} \times \mathcal{T}_{\mathcal{M}}(Y(\cdot))\right)$ by

$$
\delta_{n}:=\int_{b_{n}}^{a_{n+1}} \operatorname{dist}\left((\dot{X}(s), \dot{Y}(s)), \Phi(X(s), Y(s)) \cap\left(\mathbb{R}^{d} \times \mathcal{T}_{\mathcal{M}}(Y(s))\right)\right) d s \leq \frac{2 M}{\varepsilon} \eta_{n},
$$

where $\varepsilon$ is given in (1.2). By the Filippov approximation theorem (see [11, Theorem 3.1.6]) and also [12, Proposition 3.2]), there exists a trajectory $\left(X_{n}, Z_{n}\right)(\cdot)$ of $\Phi\left(x, Z_{n}(\cdot)\right) \cap\left(\mathbb{R}^{d} \times \mathcal{T}_{\mathcal{M}}\left(Z_{n}(\cdot)\right)\right)$ defined on the interval $\left[b_{n}, a_{n+1}\right]$ that lies in $\mathbb{R}^{d} \times \mathcal{M}$ with $Z_{n}\left(b_{n}\right)=Y\left(b_{n}\right)$ and satisfies for any , $s \in\left[b_{n}, a_{n+1}\right]$

$$
\left\|\left(X_{n}, Z_{n}\right)(s)-(X, Y)(s)\right\| \leq e^{L\left(s-b_{n}\right) / \varepsilon} \delta_{n} \leq \frac{2 M}{\varepsilon} e^{L\left(s-b_{n}\right) / \varepsilon} \eta_{n} \leq \frac{2 M}{\varepsilon} e^{L\left(a_{n+1}-b_{n}\right) / \varepsilon} \eta_{n} .
$$


Since $\left(X_{n}, Z_{n}\right)(\cdot)$ lies in $\mathbb{R}^{d} \times \mathcal{M}$ and is driven by $\Phi\left(x, Z_{n}(\cdot)\right) \cap\left(\mathbb{R}^{d} \times \mathcal{T}_{\mathcal{M}}\left(Z_{n}(\cdot)\right)\right)$ which is Lipschitz continuous, the subsolution property of $u$ implies that

$$
u\left(Z_{n}\left(b_{n}\right)\right) \leq \int_{b_{n}}^{a_{n+1}} e^{-\beta\left(s-b_{n}\right)} P \cdot \dot{X}_{n}(s) d s+e^{-\beta\left(a_{n+1}-b_{n}\right)} u\left(Z_{n}\left(a_{n+1}\right)\right) .
$$

Then by (3.10) we have

$$
\begin{aligned}
& u\left(Y\left(b_{n}\right)\right)=u\left(Z_{n}\left(b_{n}\right)\right) \\
& \leq \int_{b_{n}}^{a_{n+1}} e^{-\beta\left(s-b_{n}\right)} P \cdot \dot{X}(s) d s+\left(\int_{b_{n}}^{a_{n+1}} e^{-\beta\left(s-b_{n}\right)} d s\|P\| L \frac{2 M}{\varepsilon} e^{L\left(a_{n+1}-b_{n}\right) / \varepsilon} \eta_{n}\right) \\
& \quad+e^{-\beta\left(a_{n+1}-b_{n}\right)} u\left(Y\left(a_{n+1}\right)\right)+e^{-\beta\left(a_{n+1}-b_{n}\right)} L_{u} \cdot \frac{2 M}{\varepsilon} e^{L\left(a_{n+1}-b_{n}\right) / \varepsilon} \eta_{n} \\
& \leq \int_{b_{n}}^{a_{n+1}} e^{-\beta\left(s-b_{n}\right)} P \cdot \dot{X}(s) d s+e^{-\beta\left(a_{n+1}-b_{n}\right)} u\left(Y\left(a_{n+1}\right)\right) \\
& (3.11)+\left(\|P\| L+L_{u}\right) \frac{2 M}{\varepsilon} e^{L\left(a_{n+1}-b_{n}\right) / \varepsilon} \eta_{n},
\end{aligned}
$$

where $L_{u}$ is the Lipschitz constant of $u$. Then we deduce that

$$
\begin{aligned}
u\left(Y\left(a_{n}\right)\right) \leq & \int_{a_{n}}^{b_{n}} e^{-\beta\left(s-a_{n}\right)} P \cdot \dot{X}(s) d s+\int_{b_{n}}^{a_{n+1}} e^{-\beta\left(s-a_{n}\right)} P \cdot \dot{X}(s) d s \\
& +e^{-\beta\left(a_{n+1}-a_{n}\right)} u\left(Y\left(a_{n+1}\right)\right)+e^{-\beta\left(b_{n}-a_{n}\right)} \cdot\left(\|P\| L+L_{u}\right) \frac{2 M}{\varepsilon} e^{L\left(a_{n+1}-b_{n}\right) / \varepsilon} \eta_{n} \\
\leq & \int_{a_{n}}^{a_{n+1}} e^{-\beta\left(s-a_{n}\right)} P \cdot \dot{X}(s) d s+e^{-\beta\left(a_{n+1}-a_{n}\right)} u\left(Y\left(a_{n+1}\right)\right) \\
& +\left(\|P\| L+L_{u}\right) \frac{2 M}{\varepsilon} e^{L\left(a_{n+1}-b_{n}\right) / \varepsilon} \eta_{n} .
\end{aligned}
$$

By using (3.11) for $n=0$ and (3.12) for $n=1, \ldots, p$, we obtain

$$
\begin{aligned}
& u(Y(a)) \leq \int_{a}^{a_{1}} e^{-\beta(s-a)} P \cdot \dot{X}(s) d s+e^{-\beta\left(a_{1}-a\right)} u\left(Y\left(a_{1}\right)\right)+\left(\|P\| L+L_{u}\right) \frac{2 M}{\varepsilon} e^{L\left(a_{1}-b_{0}\right) / \varepsilon} \eta_{0} \\
\leq & \int_{a}^{a_{2}} e^{-\beta(s-a)} P \cdot \dot{X}(s) d s+e^{-\beta\left(a_{2}-a\right)} u\left(Y\left(a_{2}\right)\right)+\left(\|P\| L+L_{u}\right) \frac{2 M}{\varepsilon} e^{L\left(a_{2}-b_{0}\right) / \varepsilon}\left(\eta_{0}+\eta_{1}\right) \\
\cdots & \int_{a}^{a_{p+1}} e^{-\beta(s-a)} P \cdot \dot{X}(s) d s+e^{-\beta\left(a_{p+1}-a\right)} u\left(Y\left(a_{p+1}\right)\right)+\left(\|P\| L+L_{u}\right) \frac{2 M}{\varepsilon} e^{L\left(a_{p+1}-b_{0}\right) / \varepsilon} \sum_{n=0}^{p} \eta_{n} \\
= & \int_{a}^{b} e^{-\beta(s-a)} P \cdot \dot{X}(s) d s+e^{-\beta(b-a)} u(Y(b))+\left(\|P\| L+L_{u}\right) \frac{2 M}{\varepsilon} e^{L(b-a) / \varepsilon} \sum_{n=0}^{p} \eta_{n} .
\end{aligned}
$$

By taking $p \rightarrow+\infty$, we have $\sum_{n=0}^{p} \eta_{n}=$ meas $\left(J \backslash J_{p}\right) \rightarrow 0$ and the desired result is obtained.

Now we state the proof of Theorem 3.6.

Proof. [Proof of Theorem 3.6] Let $u$ be a subsolution of (3.1). For any trajectory $(X, Y)(\cdot) \in S[x, y]$, any $[a, b] \subset[0,+\infty)$, we want to prove that $(3.9)$ is true, i.e.

$$
u(Y(a)) \leq \int_{a}^{b} e^{-\beta(s-a)} P \cdot \dot{X}(s) d s+e^{-\beta(b-a)} u(Y(b)) .
$$


We set

$$
\Omega=\left\{\mathcal{M} \in S_{1} \cup S_{2} \cup I \mid \exists s \in[a, b] \text { such that } Y(s) \in \mathcal{M}\right\} .
$$

Note that $\Omega$ is connected since $Y(\cdot)$ is continuous.

Let $d_{\Omega}$ be the minimal dimension of the manifolds contained in $\Omega$.

Case 1: $d_{\Omega}=2$.

Then $\Omega \subset \Omega_{1} \cup \Omega_{2}$. Since $Y(\cdot)$ is continuous, then $\left.Y\right|_{[a, b]}$ lies entirely in $\Omega_{1}$ or $\Omega_{2}$. Since the dynamics $g_{i}$ of $Y(\cdot)$ is Lipschitz continuous, then the subsolution property of $u$ implies that $u$ satisfies the sub-optimality along $\left.(X, Y)\right|_{[a, b]}$, i.e. (3.9) holds true.

Case 2: $d_{\Omega}=1$.

Two cases can happen.

Case 2.1: $\Omega$ contains only one manifold

In that case, $\Omega \in I$ with dimension 1 , then the subsolution property of $u$ implies (3.9) since the dynamics $\Phi \cap\left(\mathbb{R}^{d} \times \mathcal{T}_{\Omega}\right)$ is Lipschitz continuous on $\Omega$.

\section{Case 2.2: $\Omega$ contains more than one manifold}

Let $\mathcal{M}_{1}^{\prime}, \ldots, \mathcal{M}_{p}^{\prime}$ be all the manifolds contained in $\Omega$ with dimension 1 . Then $\Omega^{\prime}:=$ $\Omega \backslash\left(\cup_{k=1}^{p} \mathcal{M}_{k}^{\prime}\right)$ contains only manifolds of dimension 2 . For any $(\bar{X}, \bar{Y}) \in S[x, y]$ with $\bar{Y}(\cdot) \subset \Omega^{\prime},(3.9)$ is satisfied (see Case 1). Then using Proposition 3.7, we get that (3.9) holds true for every trajectory $(\bar{X}, \bar{Y}) \in S[x, y]$ with $\bar{Y}(\cdot) \subset \Omega^{\prime} \cup \mathcal{M}_{1}^{\prime}$ because $\mathcal{M}_{1}^{\prime} \subset \overline{\Omega^{\prime}}$. By induction, (3.9) holds true for every $(\bar{X}, \bar{Y}) \in S[x, y]$ with $\bar{Y}(\cdot) \subset \Omega^{\prime} \cup \mathcal{M}_{1}^{\prime} \cup \cdots \cup \mathcal{M}_{p}^{\prime}=\Omega$.

Case 3: $d_{\Omega}=1$.

The arguments are quite similar to the ones of Case 2 .

Finally, to complete the proof, we remark that the sub-optimality of $u$ is proved by taking $a=0, b=h$ in (3.9).

We are now ready to prove the following comparison principle

Lemma 3.8 (Comparison principle for (3.1)). Let $u, w: \mathbb{R}^{2} \rightarrow \mathbb{R}$ be Lipschitz continuous functions. Suppose that $u$ is a subsolution of (3.1) and $w$ is a supersolution of (3.1). Then we have

$$
u(y) \leq w(y), \forall y \in \mathbb{R}^{2} .
$$

Proof. By contradiction, suppose that

$$
\sup _{y \in \mathbb{R}^{2}}\{u(y)-w(y)\}:=M>0 .
$$

Then there exists $y_{0} \in \mathbb{R}^{2}$ such that

$$
u\left(y_{0}\right)-w\left(y_{0}\right)>\frac{M}{2} .
$$

Since $w$ is a supersolution, by Theorem 3.5, $w$ satisfies the super-optimality, i.e. $\exists(\bar{X}, \bar{Y}) \in S\left[y_{0}\right]$ such that

$$
w\left(y_{0}\right) \geq e^{-\beta h} w(\bar{Y}(h))+\int_{0}^{h} e^{-\beta s} P \cdot \dot{\bar{X}}(s) d s, \forall h \geq 0 .
$$


Since $u$ is a subsolution, by Theorem 3.6, $u$ satisfies the sub-optimality, i.e.

$$
u\left(y_{0}\right) \leq e^{-\beta h} u(\bar{Y}(h))+\int_{0}^{h} e^{-\beta s} P \cdot \dot{\bar{X}}(s) d s, \forall h \geq 0 .
$$

Equations (3.15) and (3.16) leads to

$$
u\left(y_{0}\right)-w\left(y_{0}\right) \leq e^{-\beta h}(u(\bar{Y}(h))-w(\bar{Y}(h))), \forall h \geq 0 .
$$

If there exists $h_{0}>0$ such that $\bar{Y}(h)=y_{0}$, then we deduce that

$$
u\left(y_{0}\right)-w\left(y_{0}\right) \leq 0,
$$

which contradicts (3.14). Otherwise, we set $z_{h}=\bar{Y}(h)$ with $z_{h} \neq y_{0}$ and $h=\log 2 / \beta$. We then have

$$
u\left(z_{h}\right)-w\left(z_{h}\right) \geq e^{\beta h}\left(u\left(y_{0}\right)-w\left(y_{0}\right)\right)>e^{\beta h} \frac{M}{2}=M,
$$

which is a contradiction to (3.13). Thus $M \leq 0$ and the desired result holds.

We now give the proof of Theorem 3.1.

Proof. [Proof of Theorem 3.1] The fact that $w^{\beta}$ is a viscosity solution of (3.1) is a consequence of Proposition 3.4. The uniqueness is deduced from Lemma 3.8.

3.2 Proof of Theorem 1.2 Before we start the proof, we need the following stability result.

Lemma 3.9. Let $v^{\beta}$ be the viscosity solution of

$$
\beta v^{\beta}(y)+a^{\beta}+H^{E}\left(x, y, P, D v^{\beta}(y)\right)=0
$$

with $a^{\beta} \in \mathbb{R}$. Assume that there exist $\lambda \in \mathbb{R}$ and $v: \mathbb{R}^{2} \rightarrow \mathbb{R}$ such that

$$
\beta v^{\beta}+a^{\beta} \rightarrow-\lambda \text { uniformly and } v^{\beta} \rightarrow v \text { uniformly when } \beta \rightarrow 0 .
$$

Then $v$ is a viscosity solution of (1.5).

Proof. We first prove that $v$ is a subsolution. Let $y_{0} \in \mathbb{R}^{2}, \phi \in C\left(\mathbb{R}^{2}\right)$ and $\phi \in C^{1}(\overline{\mathcal{M}})$ for each $\mathcal{M} \in S_{1} \cup S_{2} \cup I$ with $y_{0} \in \overline{\mathcal{M}}$ such that $v(x)-\phi(x)$ attains a strict maximum at $y_{0}$. We want to prove that

$$
-\lambda+\sup _{(p, q) \in \Phi_{\mathcal{M}}\left(x, y_{0}\right) \cap\left(\mathbb{R}^{d} \times \mathcal{T}_{\overline{\mathcal{M}}}\left(y_{0}\right)\right)}\left\{-p \cdot P-q \cdot D_{\overline{\mathcal{M}}} \phi\left(y_{0}\right)\right\} \leq 0 .
$$

Let $\mathcal{M} \in S_{1} \cup S_{2} \cup I$ such that $y_{0} \in \overline{\mathcal{M}}$.

For any $y \in \mathbb{R}^{2}$, let $P_{\overline{\mathcal{M}}}(y)$ be the projection of $y$ on $\overline{\mathcal{M}}$, and $\operatorname{dist}(y, \overline{\mathcal{M}})$ be the distance function to $\overline{\mathcal{M}}$. Consider the penalized function $\Psi(y):=v(y)-\phi(y)-$ $C \operatorname{dist}(y, \overline{\mathcal{M}})$ with

$$
C>\left\|D v^{\beta}-D \phi\right\|
$$

We have

$$
v(y)-\phi(y)-C \operatorname{dist}(y, \overline{\mathcal{M}}) \leq v(y)-\phi(y)<v\left(y_{0}\right)-\phi\left(y_{0}\right), \quad \forall y \neq y_{0},
$$


which implies that $v(y)-\phi(y)-C \operatorname{dist}(y, \overline{\mathcal{M}})$ attains a strict maximum at $y_{0}$. Since $v^{\beta} \rightarrow v$ uniformly, $v^{\beta}-\phi+C \operatorname{dist}(y, \overline{\mathcal{M}})$ attains a local maximum at some $y_{\beta}$ with $y_{\beta} \rightarrow y_{0}$. For any $y \notin \overline{\mathcal{M}}$, we have

$$
\begin{aligned}
v^{\beta}(y)-\phi(y)-C \operatorname{dist}(y, \overline{\mathcal{M}}) & \leq v^{\beta}\left(P_{\overline{\mathcal{M}}}(y)\right)-\phi\left(P_{\overline{\mathcal{M}}}(y)\right)+\left\|D v^{\beta}-D \phi\right\| \cdot\left\|y-P_{\overline{\mathcal{M}}}(y)\right\| \\
& <v^{\beta}\left(P_{\overline{\mathcal{M}}}(y)\right)-\phi\left(P_{\overline{\mathcal{M}}}(y)\right) .
\end{aligned}
$$

Then we deduce that the maximum $y_{\beta} \in \overline{\mathcal{M}}$.

$v^{\beta}$ is the subsolution of (3.17), thus

$$
\beta v^{\beta}\left(y_{\beta}\right)+\alpha_{\beta}+\sup _{(p, q) \in \Phi_{\mathcal{M}}\left(x, y_{\beta}\right) \cap\left(\mathbb{R}^{d} \times \mathcal{T}_{\overline{\mathcal{M}}}\left(y_{\beta}\right)\right)}\left\{-p \cdot P-q \cdot D \phi\left(y_{\beta}\right)\right\} \leq 0 .
$$

We claim that

$$
\mathcal{T}_{\overline{\mathcal{M}}}\left(y_{0}\right) \subseteq \mathcal{T}_{\overline{\mathcal{M}}}\left(y_{\beta}\right)
$$

If $y_{0} \in r$-int $\overline{\mathcal{M}}$ (the relative interior of $\overline{\mathcal{M}}$ ), then $y_{\beta} \in r$-int $\overline{\mathcal{M}}$ for $\beta$ small enough. Therefore,

$$
\mathcal{T}_{\overline{\mathcal{M}}}\left(y_{\beta}\right)=\mathcal{T}_{\mathcal{M}}\left(y_{\beta}\right)=\mathcal{T}_{\mathcal{M}}\left(y_{0}\right)=\mathcal{T}_{\overline{\mathcal{M}}}\left(y_{0}\right) .
$$

If $y_{0} \in r$-bdry $\overline{\mathcal{M}}$ (the relative boundary of $\overline{\mathcal{M}}$ ), note that $y_{\beta} \rightarrow y_{0}$ and $y_{\beta} \in \overline{\mathcal{M}}$, then $y_{\beta} \in r$-bdry $\overline{\mathcal{M}}$ or $y_{\beta} \in r$-int $\overline{\mathcal{M}}$. If $y_{\beta} \in r$-bdry $\overline{\mathcal{M}}$, then

$$
\mathcal{T}_{\overline{\mathcal{M}}}\left(y_{\beta}\right)=\mathcal{T}_{\overline{\mathcal{M}}}\left(y_{0}\right) .
$$

Otherwise $y_{\beta} \in r$-int $\overline{\mathcal{M}}$, then

$$
\mathcal{T}_{\overline{\mathcal{M}}}\left(y_{0}\right) \subset \mathcal{T}_{\mathcal{M}}\left(y_{\beta}\right)=\mathcal{T}_{\overline{\mathcal{M}}}\left(y_{\beta}\right) .
$$

Finally, we conclude that (3.19) holds true.

Equations (3.18) and (3.19) implies that

$$
\beta v^{\beta}\left(y_{\beta}\right)+a^{\beta}+\sup _{(p, q) \in \Phi_{\mathcal{M}}\left(x, y_{\beta}\right) \cap\left(\mathbb{R}^{d} \times \mathcal{T}_{\overline{\mathcal{M}}}\left(y_{0}\right)\right)}\left\{-p \cdot P-q \cdot D \phi\left(y_{\beta}\right)\right\} \leq 0 .
$$

By letting $\beta \rightarrow 0$, we obtain

$$
\left.-\lambda+\sup _{(p, q) \in \Phi_{\mathcal{M}}\left(x, y_{0}\right) \cap\left(\mathbb{R}^{d} \times \mathcal{T}_{\overline{\mathcal{M}}}\left(y_{0}\right)\right)}\left\{-p \cdot P-q \cdot D_{\overline{\mathcal{M}}} \phi\left(y_{0}\right)\right)\right\} \leq 0 .
$$

Now we prove that $v$ is a supersolution. Let $\phi \in C^{1}\left(\mathbb{R}^{2}\right)$ such that $v-\phi$ attains a strict minimum at $y_{0}$. Since $v^{\beta} \rightarrow v$ uniformly, $v^{\beta}-\phi$ attains a minimum at some $y_{\beta}$ such that $y_{\beta} \rightarrow y_{0}$. Then we have

$$
\left.\beta v^{\beta}\left(y_{\beta}\right)+a^{\beta}+\sup _{(p, q) \in \Phi^{E}\left(x, y_{\beta}\right)}\left\{-p \cdot P-q \cdot D \phi\left(y_{\beta}\right)\right)\right\} \geq 0 .
$$

Since $\Phi^{E}(\cdot) \subseteq \Phi(\cdot)$, we have

$$
\left.\beta v^{\beta}\left(y_{\beta}\right)+a^{\beta}+\sup _{(p, q) \in \Phi\left(x, y_{\beta}\right)}\left\{-p \cdot P-q \cdot D \phi\left(y_{\beta}\right)\right)\right\} \geq 0 .
$$


By sending $\beta \rightarrow 0$ and the upper semi-continuity of $\Phi$, we get

$$
\left.-\lambda+\sup _{(p, q) \in \Phi\left(x, y_{0}\right)}\left\{-p \cdot P-q \cdot D \phi\left(y_{0}\right)\right)\right\} \geq 0,
$$

which, by [21, Proposition 3.5, Theorem 3.7], is equivalent to

$$
\left.-\lambda+\sup _{(p, q) \in \Phi^{E}\left(x, y_{0}\right)}\left\{-p \cdot P-q \cdot D \phi\left(y_{0}\right)\right)\right\} \geq 0 .
$$

Now we state the proof of Theorem 1.2.

Proof. [Proof of Theorem 1.2] By Theorem 3.1, given $x \in \mathbb{R}^{d}, P \in \mathbb{R}^{d}$, for each $\beta>0$, we know that the approximating problem

$$
\beta w^{\beta}(y)+H^{E}\left(x, y, P, D w^{\beta}(y)\right)=0, \text { for } y \in \mathbb{R}^{2}
$$

has a unique bounded Lipschitz continuous viscosity solution $w^{\beta}$.

Step 1: Estimate on $w^{\beta}$.

We now prove that $w^{\beta}$ is $S$-periodic. For $k \in \mathbb{Z}^{2}$, we set $\widetilde{w}^{\beta}(y):=w^{\beta}(y+k)$. It is then easy to check that $\widetilde{w}^{\beta}$ is still a solution of (3.1). Thus, by uniqueness, we get

$$
\widetilde{w}^{\beta}=w^{\beta},
$$

which implies that $w^{\beta}$ is $S$-periodic.

Since $w^{\beta}$ is uniformly Lipschitz continuous (see Propositon3.3), then $w^{\beta}$ is differentiable almost everywhere and

$$
\sup _{0<\beta<1}\left\|D w^{\beta}\right\| \leq C_{1} .
$$

Moreover, by (3.2), we get that

$$
\left\|\beta w^{\beta}\right\| \leq\|P\| M \text {. }
$$

Let $v^{\beta}=w^{\beta}-\min _{S} w^{\beta}$. Since $w^{\beta}$ is continuous and periodic, there exists $y_{0} \in S$ such that $v^{\beta}=w^{\beta}-w^{\beta}\left(x_{0}\right)$. Then

$$
\left\|v^{\beta}\right\| \leq 2 \sqrt{2}\left\|D w^{\beta}\right\| \leq 2 \sqrt{2} C_{1}, \quad D v^{\beta}=D w^{\beta} .
$$

Using the fact that $w^{\beta}$ is a viscosity solution of (3.1), we get that $v^{\beta}$ is a viscosity solution of

$$
\beta v^{\beta}(y)+H^{E}\left(x, y, P, D v^{\beta}(y)\right)=-\min _{S}\left(\beta w^{\beta}\right), \forall y \in \mathbb{R}^{2} .
$$

\section{Step 2: Passing to the limit}

Using (3.20), (3.21) and Arzela-Ascoli Theorem, up to a subsequence, we get

$$
v^{\beta} \rightarrow v \text { uniformly on } \mathbb{R}^{2} \text { and } \min _{S}\left(\beta w^{\beta}\right) \rightarrow-\lambda
$$

for some $v$ Lipschitz continuous and $S$-periodic and $\lambda \in \mathbb{R}$. Moreover, since $v^{\beta}$ is uniformly bounded (see (3.21)), we get

$$
\beta v^{\beta} \rightarrow 0 \text { uniformly on } \mathbb{R}^{2} \text {. }
$$


Then by Lemma 3.9, we deduce that

$$
H^{E}(x, y, P, D v(y))=\lambda \text {. }
$$

\section{Step 3: Uniqueness of $\lambda$}

Suppose that there exists $\left(v_{1}, \lambda_{1}\right)$ and $\left(v_{2}, \lambda_{2}\right)$ solutions of the cell problem (1.5) with $\lambda_{1} \neq \lambda_{2}$. Assume without lost of generality that $\lambda_{1}<\lambda_{2}$. Note that $v_{1}, v_{2}$ are both continuous and periodic, thus they are bounded. By adding a suitable constant to $v_{1}$, we may assume that $v_{1}>v_{2}$.

Since $\lambda_{1}<\frac{\lambda_{1}+\lambda_{2}}{2}<\lambda_{2}, v_{1}, v_{2}$ are bounded, we deduce that for $\varepsilon$ small enough, $v_{1}, v_{2}$ are respectively subsolution and supersolution of

$$
\varepsilon v+H^{E}(x, y, P, D v)=\frac{\lambda_{1}+\lambda_{2}}{2} .
$$

Using the comparison principle for the equation (3.1), we obtain $v_{1} \leq v_{2}$ which is a contradiction.

\section{Properties of the effective Hamiltonian For every} $x \in \mathbb{R}^{d}, P \in \mathbb{R}^{d}$, we denote by $\bar{H}(x, P)$ the unique constant such that there exists a periodic solution of (1.5).

Proposition 4.1. $\bar{H}(\cdot, \cdot)$ is Lipschitz continuous.

Proof. Let $x_{1}, x_{2} \in \mathbb{R}^{d}$ and $P_{1}, P_{2} \in \mathbb{R}^{2}$. For each $\beta>0$, suppose that $w_{i}^{\beta}, i=1,2$ is a solution of

$$
\beta w_{i}^{\beta}(y)+H^{E}\left(x_{i}, y, P_{i}, D w_{i}^{\beta}\right)=0,
$$

For any $y \in \mathbb{R}^{2}, q \in \mathbb{R}^{2}$, by the Lipschitz continuity of $H^{E}(\cdot, y, \cdot, q)$, there exists $C>0$ such that

$$
H^{E}\left(x_{2}, y, P_{2}, q\right) \leq H^{E}\left(x_{1}, y, P_{1}, q\right)+C\left(\left\|x_{1}-x_{2}\right\|+\left\|P_{1}-P_{2}\right\|\right) .
$$

Then we deduce that $w_{1}^{\beta}-\frac{C}{\beta}\left(\left\|x_{1}-x_{2}\right\|+\left\|P_{1}-P_{2}\right\|\right)$ is a subsolution of

$$
\beta w^{\beta}+H^{E}\left(x_{2}, y, P_{2}, D w^{\beta}\right)=0 .
$$

By the comparison principle for (3.1), we get

$$
w_{1}^{\beta}-\frac{C}{\beta}\left(\left\|x_{1}-x_{2}\right\|+\left\|P_{1}-P_{2}\right\|\right) \leq w_{2}^{\beta},
$$

i.e.

$$
\beta w_{1}^{\beta}-\beta w_{2}^{\beta} \leq C\left(\left\|x_{1}-x_{2}\right\|+\left\|P_{1}-P_{2}\right\|\right) .
$$

Letting $\beta \rightarrow 0$ leads to

$$
\bar{H}\left(x_{1}, P_{1}\right)-\bar{H}\left(x_{2}, P_{2}\right) \leq C\left(\left\|x_{1}-x_{2}\right\|+\left\|P_{1}-P_{2}\right\|\right) .
$$

Exchanging the role of $\left(x_{1}, P_{1}\right)$ and $\left(x_{2}, P_{2}\right)$, we conclude that

$$
\left|\bar{H}\left(x_{1}, P_{1}\right)-\bar{H}\left(x_{2}, P_{2}\right)\right| \leq C\left(\left\|x_{1}-x_{2}\right\|+\left\|P_{1}-P_{2}\right\|\right),
$$

which implies the Lipschitz continuity of $\bar{H}(\cdot, \cdot)$. 
As studied in $[9,1]$, the effective Hamiltonian $\bar{H}$ can be evaluated as the optimal average cost of an ergodic control problem in the $y$ variable.

Proposition 4.2. Given $x \in \mathbb{R}^{d}, P \in \mathbb{R}^{d}$,

$$
\bar{H}(x, P)=\lim _{t \rightarrow+\infty} \sup _{(X, Y) \in S[x, y]}\left\{-\frac{1}{t} P \cdot(X(t)-x)\right\},
$$

for any $y \in \mathbb{R}^{2}$.

Proof. This result is quite similar to the formula (10) obtained in [1]. Here we give a sketch of the proof. Consider the value function

$$
v(t, y)=\inf _{(X, Y) \in S[x, y]}\{P \cdot(X(t)-x)\} .
$$

Then $v$ solves the HJB equation

$$
\partial_{t} v(t, y)+H^{E}\left(x, y, P, D_{y} v(t, y)\right)=0 \text { on }(0,+\infty) \times \mathbb{R}^{2},
$$

where $x, P$ are fixed, and the initial condition $v(0, \cdot) \equiv 0$. Let $w(\cdot)$ be a solution of the cell problem (1.5) with $\lambda=\bar{H}(x, P)$, then $w(y)-t \bar{H}(x, P)$ is a solution of the same Cauchy problem but with a different initial condition. Note that the HJB equation above is the same type as (1.4), the comparison result Theorem 2.7 implies that $v(t, y)-w(y)+t \bar{H}(x, P)$ is bounded by $\|w\|_{\infty}$. Since $w$ is bounded, $-v(t, y) / t \rightarrow$ $\bar{H}(x, P)$ as $t \rightarrow+\infty$, uniformly in $y$.

Remark 4.3. If we consider the same case as in [7] where the controls acting on the slow variable $X$ and and fast variable $Y$ are separated, more precisely given $A, B$ two independent control sets,

$$
f=f(x, y, a), a \in A, g_{i}=g_{i}(x, y, b), b \in B, i=1,2 .
$$

Let $H_{1}: \mathbb{R}^{2} \times \mathbb{R}^{2} \times \mathbb{R}^{d} \rightarrow \mathbb{R}$ defined by

$$
H_{1}(x, y, P)=\sup _{a \in A}\{-P \cdot f(x, y, a)\} .
$$

Then the effective Hamiltonian satisfies the following formula:

$$
\bar{H}(x, P)=\max _{y \in \mathbb{R}^{2}} H_{1}(x, y, P), \forall x \in \mathbb{R}^{d}, P \in \mathbb{R}^{d} .
$$

This is the same formula (12) obtained in [1]. It is proved through the formula (4.1) and the controllability assumption on the fast variable $Y$.

\section{Proof of Theorem 1.3 We define}

$$
\bar{u}(t, x)=\limsup _{t, x, \varepsilon} \sup _{y \in \mathbb{R}^{2}} u^{\varepsilon}(t, x, y) \quad \text { and } \quad \underline{u}(t, x)=\lim _{t, x, \varepsilon} \inf _{y \in \mathbb{R}^{2}} u^{\varepsilon}(t, x, y) .
$$

The proof is divided into several steps.

Step $1: \bar{u}$ is subsolution of (1.6).

Let $\phi \in C^{1}\left((0, T) \times \mathbb{R}^{d}\right)$ such that $\bar{u}-\phi$ has a strict local maximum at $\left(t_{0}, x_{0}\right)$. We want to prove that

$$
-\phi_{t}\left(t_{0}, x_{0}, y_{0}\right)+\bar{H}\left(x_{0}, D \phi\left(t_{0}, x_{0}\right)\right) \leq 0
$$


We assume by contradiction that

$$
-\phi_{t}\left(t_{0}, x_{0}\right)+\bar{H}\left(x_{0}, D \phi\left(t_{0}, x_{0}\right)\right)=\theta>0 .
$$

We set $P:=D \phi\left(t_{0}, x_{0}\right)$ and let $v$ be a periodic Lipschitz continuous viscosity solution of the cell problem

$$
-\bar{H}\left(x_{0}, P\right)+H^{E}\left(x_{0}, y, P, D v(y)\right)=0 .
$$

We use the perturbed test function introduced by Evans. For any $\varepsilon>0$, we define $\phi^{\varepsilon}(t, x, y)=\phi(t, x)+\varepsilon v(y)$. We want to prove that $\phi^{\varepsilon}$ is a supersolution of (1.4) in $B\left(\left(t_{0}, x_{0}\right), r\right) \times \mathbb{R}^{2}$ for $r>0$ small enough. Let $\psi \in C^{1}\left((0, T) \times \mathbb{R}^{d} \times \mathbb{R}^{2}\right)$ such that $\phi^{\varepsilon}-\psi$ attains a minimum at $\left(t_{1}, x_{1}, y_{1}\right) \in B\left(\left(t_{0}, x_{0}\right), r\right) \times \mathbb{R}^{2}$. Then

$$
\phi^{\varepsilon}\left(t_{1}, x_{1}, y_{1}\right)-\psi\left(t_{1}, x_{1}, y_{1}\right) \leq \phi^{\varepsilon}(t, x, y)-\psi(t, x, y) .
$$

This implies that

$$
v\left(y_{1}\right)-\Gamma\left(y_{1}\right) \leq v(y)-\Gamma(y)
$$

where $\Gamma(y)=\frac{1}{\varepsilon}\left[\psi\left(t_{1}, x_{1}, y\right)-\phi\left(t_{1}, x_{1}\right)\right]$. We deduce that $v(y)-\Gamma(y)$ attains a minimum at $y_{1}$, then

$$
-\bar{H}\left(x_{0}, P\right)+H^{E}\left(x_{0}, y_{1}, P, D \Gamma\left(y_{1}\right)\right) \geq 0,
$$

i.e.

$$
-\phi_{t}\left(t_{0}, x_{0}\right)-\theta+H^{E}\left(x_{0}, y_{1}, D \phi\left(t_{0}, x_{0}\right), \frac{1}{\varepsilon} D_{y} \psi\left(t_{1}, x_{1}, y_{1}\right)\right) \geq 0 .
$$

We then deduce that

$$
\begin{aligned}
& -\phi_{t}\left(t_{1}, x_{1}\right)+H^{E}\left(x_{1}, y_{1}, D \phi\left(t_{1}, x_{1}\right), \frac{1}{\varepsilon} D_{y} \psi\left(t_{1}, x_{1}, y_{1}\right)\right) \geq \phi_{t}\left(t_{0}, x_{0}\right)-\phi_{t}\left(t_{1}, x_{1}\right)-\theta \\
& +H^{E}\left(x_{1}, y_{1}, D \phi\left(t_{1}, x_{1}\right), \frac{1}{\varepsilon} D_{y} \psi\left(t_{1}, x_{1}, y_{1}\right)\right)-H^{E}\left(x_{0}, y_{1}, D \phi\left(t_{0}, x_{0}\right), \frac{1}{\varepsilon} D_{y} \psi\left(t_{1}, x_{1}, y_{1}\right)\right) .
\end{aligned}
$$

Since $\phi \in C^{1}\left((0, T) \times \mathbb{R}^{d}\right)$ and $H^{E}(\cdot, y, \cdot, q)$ is continuous, we have for $r>0$ small enough

$$
-\phi_{t}\left(t_{1}, x_{1}\right)+H^{E}\left(x_{1}, y_{1}, D \phi\left(t_{1}, x_{1}\right), \frac{1}{\varepsilon} D_{y} \psi\left(t_{1}, x_{1}, y_{1}\right)\right) \geq \frac{\theta}{2} .
$$

Note that $v(\cdot)$ is independent on $t$ and $x$, the application $t \mapsto \phi\left(t, x_{1}\right)-\psi\left(t, x_{1}, y_{1}\right)$ is $C^{1}$ and attains a minimum at $t_{1}$ and the application $x \mapsto \phi\left(t_{1}, x\right)-\psi\left(t_{1}, x, y_{1}\right)$ is $C^{1}$ and attains a minimum at $x_{1}$, we obtain

$$
\phi_{t}\left(t_{1}, x_{1}\right)=\psi_{t}\left(t_{1}, x_{1}, y_{1}\right), D \phi\left(t_{1}, x_{1}\right)=D_{x} \psi\left(t_{1}, x_{1}, y_{1}\right) .
$$

We conclude that

$$
-\psi_{t}\left(t_{1}, x_{1}, y_{1}\right)+H^{E}\left(x_{1}, y_{1}, D_{x} \psi\left(t_{1}, x_{1}, y_{1}\right), \frac{1}{\varepsilon} D_{y} \psi\left(t_{1}, x_{1}, y_{1}\right)\right) \geq \frac{\theta}{2},
$$

which implies that $\phi^{\varepsilon}$ is a supersolution of (1.4). Then by Theorem 2.7, we have

$$
\max _{B\left(\left(t_{0}, x_{0}\right), r\right) \times \mathbb{R}^{2}}\left\{u^{\varepsilon}(t, x, y)-\phi^{\varepsilon}(t, x, y)\right\} \leq \max _{\partial B\left(\left(t_{0}, x_{0}\right), r\right) \times \mathbb{R}^{2}}\left\{u^{\varepsilon}(t, x, y)-\phi^{\varepsilon}(t, x, y)\right\} .
$$


Then we deduce that

$$
\max _{(t, x) \in B\left(\left(t_{0}, x_{0}\right), r\right)}\{\bar{u}(t, x)-\phi(t, x)\} \leq \max _{(t, x) \in \partial B\left(\left(t_{0}, x_{0}\right), r\right)}\{\bar{u}(t, x)-\phi(t, x)\},
$$

which contradicts the fact that $\left(t_{0}, x_{0}\right)$ is a local strict maximum of $\bar{u}-\phi$.

Step $2: \bar{u}$ is a supersolution of (1.6).

The proof is very similar. The main difference is to check that $\phi^{\varepsilon}$ is a subsolution. By contradiction, assume that there exists $\phi \in C^{1}\left((0, T) \times \mathbb{R}^{d}\right)$ such that $\bar{u}-\phi$ has a strict local minimum at $\left(t_{0}, x_{0}\right)$.and such that

$$
-\phi_{t}\left(t_{0}, x_{0}\right)+\bar{H}\left(x_{0}, D \phi\left(t_{0}, x_{0}\right)\right)=-\theta<0 .
$$

We set $P:=D \phi\left(t_{0}, x_{0}\right)$ and let $v$ be a periodic Lipschitz continuous viscosity solution of the cell problem

$$
-\bar{H}\left(x_{0}, P\right)+H^{E}\left(x_{0}, y, P, D v(y)\right)=0 .
$$

For any $\varepsilon>0$, we define $\phi^{\varepsilon}(t, x, y)=\phi(t, x)+\varepsilon v(y)$. We want to prove that $\phi^{\varepsilon}$ is a supersolution of (1.4) in $B\left(\left(t_{0}, x_{0}\right), r\right) \times \mathbb{R}^{2}$ for $r>0$ small enough. Let $\psi:(0, T) \times$ $\mathbb{R}^{d} \times \mathbb{R}^{2} \rightarrow \mathbb{R}$ be continuous with $\left.\psi\right|_{(0, T) \times \mathbb{R}^{d} \times \overline{\mathcal{M}}}$ being $C^{1}$ for each $\mathcal{M} \in S_{1} \cup S_{2} \cup I$ with $y_{1} \in \overline{\mathcal{M}}$ such that $u-\psi$ attains a local maximum at $\left(t_{1}, x_{1}, y_{1}\right) \in B\left(\left(t_{0}, x_{0}\right), r\right) \times \mathbb{R}^{2}$. As in the previous tep, we deduce that $v-\Gamma$ reaches a maximum at $y_{1}$ where

$$
\Gamma(y)=\frac{1}{\varepsilon}\left[\psi\left(t_{1}, x_{1}, y\right)-\phi\left(t_{1}, x_{1}\right)\right] .
$$

Then

$$
\left.-\bar{H}\left(x_{0}, P\right)+\sup _{(p, q) \in \Phi_{\mathcal{M}}\left(x_{0}, y_{1}\right) \cap\left(\mathbb{R}^{d} \times \mathcal{T}_{\overline{\mathcal{M}}}\left(y_{1}\right)\right)}\left\{-p \cdot P-q \cdot D_{\overline{\mathcal{M}}} \psi\left(t_{1}, x_{1}, y_{1}\right)\right)\right\} \leq 0 .
$$

i.e.

$\left.-\phi_{t}\left(t_{0}, x_{0}\right)+\theta+\sup _{(p, q) \in \Phi_{\mathcal{M}}\left(x_{0}, y_{1}\right) \cap\left(\mathbb{R}^{d} \times \mathcal{T}_{\overline{\mathcal{M}}}\left(y_{1}\right)\right)}\left\{-p \cdot D \phi\left(t_{0}, x_{0}\right)-q \cdot D_{\overline{\mathcal{M}}} \psi\left(t_{1}, x_{1}, y_{1}\right)\right)\right\} \leq 0$.

Then we deduce that

$$
\begin{aligned}
& \left.-\phi_{t}\left(t_{1}, x_{1}\right)+\operatorname{sp}_{(p, q) \in \Phi_{\mathcal{M}}\left(x_{1}, y_{1}\right) \cap\left(\mathbb{R}^{d} \times \mathcal{T}_{\overline{\mathcal{M}}}\left(y_{1}\right)\right)}\left\{-p \cdot D \phi\left(t_{1}, x_{1}\right)-q \cdot D_{\overline{\mathcal{M}}} \psi\left(t_{1}, x_{1}, y_{1}\right)\right)\right\} \\
\leq & \phi_{t}\left(t_{0}, x_{0}\right)-\phi_{t}\left(t_{1}, x_{1}\right) \\
& \left.+\sup _{(p, q) \in \Phi_{\mathcal{M}}\left(x_{1}, y_{1}\right) \cap\left(\mathbb{R}^{d} \times \mathcal{T}_{\overline{\mathcal{M}}}\left(y_{1}\right)\right)}\left\{-p \cdot D \phi\left(t_{1}, x_{1}\right)-q \cdot D_{\overline{\mathcal{M}}} \psi\left(t_{1}, x_{1}, y_{1}\right)\right)\right\} \\
& \left.-\sup _{(p, q) \in \Phi_{\mathcal{M}}\left(x_{0}, y_{1}\right) \cap\left(\mathbb{R}^{d} \times \mathcal{T}_{\overline{\mathcal{M}}}\left(y_{1}\right)\right)}\left\{-p \cdot D \phi\left(t_{0}, x_{0}\right)-q \cdot D_{\overline{\mathcal{M}}} \psi\left(t_{1}, x_{1}, y_{1}\right)\right)\right\}-\theta .
\end{aligned}
$$

Since $\phi \in C^{1}\left((0, T) \times \mathbb{R}^{d}\right)$ and $\Phi_{\mathcal{M}}\left(\cdot, y_{1}\right) \cap\left(\mathbb{R}^{d} \times \mathcal{T}_{\overline{\mathcal{M}}}\left(y_{1}\right)\right)$ are continuous, we have for $r>0$ small enough

$$
\left.-\phi_{t}\left(t_{1}, x_{1}\right)+\sup _{(p, q) \in \Phi_{\mathcal{M}}\left(x_{1}, y_{1}\right) \cap\left(\mathbb{R}^{d} \times \mathcal{T}_{\overline{\mathcal{M}}}\left(y_{1}\right)\right)}\left\{-p \cdot D \phi\left(t_{1}, x_{1}\right)-q \cdot D_{\overline{\mathcal{M}}} \psi\left(t_{1}, x_{1}, y_{1}\right)\right)\right\} \leq-\frac{\theta}{2} .
$$

Using that

$$
\phi_{t}\left(t_{1}, x_{1}\right)=\psi_{t}\left(t_{1}, x_{1}, y_{1}\right), D \phi\left(t_{1}, x_{1}\right)=D_{x} \psi\left(t_{1}, x_{1}, y_{1}\right)
$$


we conclude that

$\left.-\psi_{t}\left(t_{1}, x_{1}, y_{1}\right)+\sup _{(p, q) \in \Phi_{\mathcal{M}}\left(x_{1}, y_{1}\right) \cap\left(\mathbb{R}^{d} \times \mathcal{T}_{\overline{\mathcal{M}}}\left(y_{1}\right)\right)}\left\{-p \cdot D \phi\left(t_{1}, x_{1}\right)-q \cdot D_{\overline{\mathcal{M}}} \psi\left(t_{1}, x_{1}, y_{1}\right)\right)\right\} \leq-\frac{\theta}{2}$,

which implies that $\phi^{\varepsilon}$ is a subsolution of (1.4). We then get a contradiction as in the previous step.

\section{Step 3: Terminal condition}

Now we check the terminal condition. We set

$$
\underline{\varphi}(x):=\inf _{y \in \mathbb{R}^{2}} \varphi(x, y) .
$$

The Lipschitz continuity of $\varphi$ implies that $\underline{\varphi}$ is Lipschitz continuous. Since $u^{\varepsilon}(T, x, y)=$ $\varphi(x, y)$, we have

$$
\inf _{y \in \mathbb{R}^{2}} u^{\varepsilon}(T, x, y)=\underline{\varphi}(x) .
$$

Then we deduce that $\underline{u}(T, x)=\underline{\varphi}(x)$.

On the other hand, for any $t \in[0, T], x \in \mathbb{R}^{d}$ and $y \in S\left(=(-1,1)^{2}\right)$,

$$
\begin{aligned}
u^{\varepsilon}(t, x, y) & =\inf _{(X, Y) \in S_{[t, T]}^{\varepsilon}(x, y)}\{\varphi(X(T), Y(T))\} \\
& \leq \inf _{(X, Y) \in S_{[t, T]}^{\varepsilon}(x, y)}\left\{\varphi(x, Y(T))+L_{\varphi}\|x-X(T)\|\right\} \\
& \leq \inf _{(X, Y) \in S_{[t, T]}^{\varepsilon}(x, y)} \varphi(x, Y(T))+M L_{\varphi}(T-t) .
\end{aligned}
$$

By the controllability assumption (H2)(iii) for $g_{i}$, we set that for any $x^{\prime} \in \mathbb{R}^{d}, Y(\cdot)$ such that $\left(x^{\prime}, Y\right) \in S\left[x^{\prime}, y\right]$,

$$
\inf \varphi\left(x^{\prime}, Y(T)\right)=\inf _{y \in S} \varphi\left(x^{\prime}, y\right)=\underline{\varphi}\left(x^{\prime}\right), \text { for } T \geq t+\frac{2 \sqrt{2} \varepsilon}{r_{0}},
$$

where we have used that $S \subset B(0, \sqrt{2})$.

Then for any $t<T$ we can restrict $\varepsilon<r_{0}(T-t) /(2 \sqrt{2})$ and get

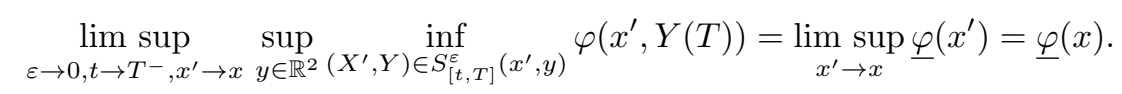

Therefore,

$$
\bar{u}(T, x) \leq \underline{\varphi}(x)+\limsup _{t \rightarrow T^{-}} M L_{\varphi}(T-t)=\underline{\varphi}(x) .
$$

We conclude that

$$
\bar{u}(T, x) \leq \underline{\varphi}(x)=\underline{u}(T, x) .
$$

\section{Step 4 : Conclusion}

Since $\bar{u}$ is a subsolution of (1.6) and $\underline{u}$ is a supersolution of (1.6), by (5.3) and the comparison principle for (1.6) we have

$$
\bar{u}(t, x) \leq \underline{u}(t, x), \text { for }(t, x) \in(0, T) \times \mathbb{R}^{d},
$$


which gives

$$
\bar{u}=\underline{u}=u \text { in }(0, T) \times \mathbb{R}^{d},
$$

and implies the convergence of $u^{\varepsilon}$ to $u$ which is the viscosity solution of (1.6).

ACKNOWLEDGMENTS The authors would like to thanks H. Zidani for fruitful discussions during the preparation of the article.

\section{REFERENCES}

[1] O. Alvarez, AND M. BARDI, Viscosity solutions methods for singular perturbations in deterministic and stochastic control, SIAM J. Control Optim., vol. 40, No. 4, pp. 11591188.

[2] O. Alvarez, AND M. BARdi, Singular perturbations of nonlinear degenerate parabolic PDEs: a general convergence result, Arch. Ration. Mech. Anal., vol.170, No. 1, pp. 17-61.

[3] J.-P. Aubin AND A. Cellina, Differential inclusions, vol. 264 of Comprehensive studies in mathematics, Springer, Berlin, Heidelberg, New York, Tokyo, 1984.

[4] Y. Achdou, F. Camilli, A. Cutri AND N. Tchou, Hamilton-Jacobi equations on networks, preprint in Nonlinear Differ. Equ. Appl., 2013.

[5] M. Amar, G. Crasta AND A. Malusa, On the Finsler Metrics Obtained as Limits of Chessboard Structures, Adv. Calc. Var., vol. 2 (2009), pp. 321-360.

[6] A. Bensoussan, Perturbation Methods in Optimal control, Wiley/Gauthiers-Villars, Chichester, U.K.,1988.

[7] F. Bagagiolo, AND M. Bardi, Singular perturbation of a finite horizon problem with state-space constraints, SIAM J. Control Optim., 36 (1998), pp. 2040-2060.

[8] G. Barles, A. Briani AND E. Chasseigne, A Bellman approach for two-domains optimal control problems in $\mathbb{R}^{N}$, preprint in ESAIM Control Optim. Calc. Var., 2013.

[9] M. Bardi AND I. Capuzzo-Dolcetta, Optimal Control and Viscosity Solutions of Hamilton-Jacobi-Bellman Equations, Systems and Control: Foundations and Applications, Birkhäuser, Boston, 1997.

[10] R.C. Barnard AND P.R. Wolenski, Flow invariance on stratified domains, preprint in Set-Valued and Variational Analysis, 2013. DOI:10.1007/s11228-013-0230-y.

[11] F.H. Clarke, Optimization and Nonsmooth Analysis, Society for Industrial Mathematics, 1990.

[12] F.H. Clarke, Yu.S. Ledyaev, R.J. Stern AND P.R. Wolenski, Nonsmooth Analysis and Control Theory, Graduate Texts in Mathematics 178, Springer-Verlag, New York.

[13] L.C. Evans, Periodic homogenisation of certain fully nonlinear partial differential equations, Proceedings of the Royal Society of Edinburgh, 120A (1992), pp. 245-265.

[14] H. Frankowska, Lower semicontinuous solutions of Hamilton-Jacobi-Bellman equations, SIAM J. Control Optim., 31 (1993), No. 1, pp. 257-272.

[15] V. Gaitsgory, Suboptimization of singularly perturbed control systems, SIAM J. Control Optim., 30 (1992), pp. 1228-1249.

[16] C. Imbert, R. Monneau AND H. Zidani, A Hamilton-Jacobi approach to junction problems and application to traffic flows, ESAIM Control Optim. Calc. Var. 19, 01 (2013) pp. 129166. DOI: $10.1051 / \mathrm{cocv} / 2012002$.

[17] P. Koкотović, H. Khalil AND J. O'Reilly, Singular Perturbation Methods in Control: Analysis and Design, Academic Press, London, 1986.

[18] P.L. Lions, G. Papanicolaou, AND S. Varadhan, Homogenization of Hamilton-Jacobi equations, unpublished.

[19] A.m. Oberman, R. Takei, AND A. Vladimirsky, Homogenization of metric HamiltonJacobi equations, Multiscale Modeling and Simulation 8/1: 269-295 (2009).

[20] Z. Rao, A. Siconolfi AND H. Zidani, Transmission conditions on interfaces for HamiltonJacobi-Bellman equations, in preparation.

[21] Z. RAO AND H. ZidANI, Hamilton-Jacobi-Bellman equations on multi-domains, Control and Optimization with PDE Constraints, International Series of Numerical Mathematics, vol. 164 (2013), Birkhäuser Basel. 